\title{
How supply chain coordination affects the environment: a carbon footprint perspective
}

\author{
Ayşegül Toptal $^{1}$ - Bilgesu Çetinkaya ${ }^{2}$
}

Published online: 18 April 2015

C) Springer Science+Business Media New York 2015

\begin{abstract}
Environmental responsibility has become an important part of doing business. Government regulations and customers' increased awareness of environmental issues are pushing supply chain entities to reduce the negative influence of their operations on the environment. In today's world, companies must assume joint responsibility with their suppliers for the environmental impact of their actions. In this paper, we study coordination between a buyer and a vendor under the existence of two emission regulation policies: cap-and-trade and tax. We investigate the impact of decentralized and centralized replenishment decisions on total carbon emissions. The buyer in this system faces a deterministic and constant demand rate for a single product in the infinite horizon. The vendor produces at a finite rate and makes deliveries to the buyer on a lot-for-lot basis. Both the buyer and the vendor aim to minimize their average annual costs resulting from replenishment set-ups and inventory holding. We provide decentralized and centralized models for the buyer and the vendor to determine their ordering/production lot sizes under each policy. We compare the solutions due to independent and joint decision-making both analytically and numerically. Finally, we arrive at coordination mechanisms for this system to increase its profitability. However, we show that even though such coordination mechanisms help the buyer and the vendor decrease their costs without violating emission regulations, the cost minimizing solution may result in increased carbon emission under certain circumstances.
\end{abstract}

Keywords Environmental regulations $\cdot$ Buyer-vendor coordination $\cdot$ Supply chains

\section{Introduction and literature}

Since the Industrial Revolution, the levels of greenhouse gases in the atmosphere have increased due to human activities. The World Meteorological Organization (WMO) (2013a)

\footnotetext{
$\bowtie$ Ayşegül Toptal

toptal@bilkent.edu.tr

1 Industrial Engineering Department, Bilkent University, 06800 Ankara, Turkey

2 Product Management Directorate, Arçelik Inc., Karaağaç Street No: 2-6, 34445 Sütlüce, Istanbul, Turkey
} 
reports that the atmospheric concentrations of the greenhouse gases exhibited an upward and accelerating trend and reached a record high in 2012. Greenhouse gases slow or prevent the loss of heat to space, which increases the temperature of Earth's surface, leading to global warming. Greenhouse gases are emitted as a result of the activities of energy industries, transportation, residential and commercial activities, manufacturing, construction, industrial processes, and agriculture. Carbon dioxide $\left(\mathrm{CO}_{2}\right)$ is the main greenhouse gas emitted as a result of the human activities; it is responsible for $85 \%$ of the increase in global warming. The effect of $\mathrm{CO}_{2}$ is followed by methane $\left(\mathrm{CH}_{4}\right.$, ) and then nitrous oxide $\left(\mathrm{N}_{2} \mathrm{O}\right)$ (WMO 2013b). To decrease greenhouse gases (particularly $\mathrm{CO}_{2}$ ) emissions, policy makers and international organizations have proposed agreements and regulations. In this paper, we study the independent and coordinated inventory replenishment decisions of a buyer and a vendor under two different emission regulation policies (i.e., cap-and-trade and tax), and investigate the impact of coordinated decisions on the environment.

Under a cap-and-trade mechanism, the government sets a fixed value for the maximum amount of carbon that can be emitted in each period (i.e., the cap) and firms are free to buy or sell allowances in trading markets. Emission trading systems (ETSs) are currently implemented in the EU (EU ETS), Australia, New Zealand (NZ ETS), Northeastern United States, and Tokyo (Tokyo ETS), as well as in other countries (see the International Emissions Trading Association's web site International Emissions Trading Assosication 2013). The carbon tax mechanism puts a price on each tonne of greenhouse gas (e.g., $\mathrm{CO}_{2}$ ) emitted. According to the Center for Climate and Energy Solutions (2013), Finland, the Netherlands, Norway, Sweden, the UK and Australia are among countries that have implemented a carbon tax.

Issues related to environmental policies, such as regulation design and the effect of a domestic environmental policy on international trade or social welfare, and others, have been widely investigated in environmental economics since the late 1960s (Cropper and Oates 1992). In contrast, the literature in operations management that considers environmental concerns is fairly new, and focuses on tactical or operational planning decisions. Some of these studies do not particularly assume the existence of environmental regulations; rather, they optimize an objective function that incorporates terms dependent on environmental performance metrics (e.g., Bonney and Jaber 2011; Bouchery et al. 2011; Chan et al. 2013; Saadany et al. 2011), or investigate the impact of supply chain members' greening efforts on their profitability in different settings with environmentally conscious consumers (e.g., Liu et al. 2012; Swami and Shah 2013). Another group of papers studies problems such as singleitem inventory replenishment, product mix, or green investment decisions, while considering a specific environmental regulation policy (e.g., Benjaafar et al. 2013; Chen et al. 2013; Dong et al. 2014; Du et al. 2011; Hoen et al. 2014; Hua et al. 2011; Jaber et al. 2013; Krass et al. 2013; Letmathe and Balakrishnan 2005; Song and Leng 2012; Toptal et al. 2014; Zhang and Xu 2013). Of these papers, Benjaafar et al. (2013), Dong et al. (2014), Du et al. (2011), Krass et al. (2013) and Jaber et al. (2013) model supply chain problems in multi-echelon settings, as our study does.

Benjaafar et al. (2013) propose an integrated model to solve the joint lot-sizing decisions of multiple firms subject to emission caps. Krass et al. (2013) consider a two-echelon system in which the upper echelon is the policy maker who maximizes social welfare and the lower echelon is a firm that maximizes its profits. In this setting, the authors analyze a Stackelberg game under three different environmental polices: tax-only, tax-and-subsidy, and a joint policy that also includes rebates given to consumers who buy products manufactured with green technologies. The policy maker, as the Stackelberg leader, decides the parameters of the different policies and the firm chooses the emission-reducing technology and the selling price. Jaber et al. (2013) investigate the impact of coordination on some environmental mea- 
sures in a manufacturer-retailer setting. In this setting, manufacturer is the only party who is subject to an environmental policy. Manufacturer's emissions due to his/her production rate are penalized with a per-unit emission cost and a fixed penalty if the total amount of emissions exceeds a limit. This combination policy allows for the modeling of a tax policy and a variant of the cap-and-trade policy. Specifically, as in cap-and-trade policy, a cost is incurred when an upper bound on the emissions is exceeded. However, unlike the typical cap-and-trade policy, it does not allow for the possibility of gains when the emissions are under the upper bound. In analyzing the impact of coordination on the environment, the authors look into how the solution to the integrated problem changes the sum of emissions and penalty costs in comparison to independently-made decisions of the parties. They observe over a set of examples that total system costs reduce with no change in the sum of emissions and penalty costs.

As opposed to Benjaafar et al. (2013), Krass et al. (2013) and Jaber et al. (2013), the studies of Dong et al. (2014) and Du et al. (2011) consider stochastic demand environments. Du et al. (2011) analyze a two-echelon system in which the upper echelon, as the permit supplier, decides the permit selling price, and the lower echelon, as the manufacturer, decides his/her production quantity. In this system, if the manufacturer needs more carbon allowance, he/she purchases it from the permit supplier, but does not have the option to sell if he/she has excess carbon allowance. In the manufacturer-retailer setting considered by Dong et al. (2014), the retailer decides the order quantity in response to the manufacturer's decision regarding the sustainability investment. The manufacturer in this setting is subject to a capand-trade policy, and both the selling and purchasing prices of the unit carbon allowance are the same. The authors also examine some of the traditional contracting mechanisms and show that revenue-sharing contracts can be used for coordinating this supply chain system.

In this paper, we consider a buyer-vendor system with deterministic and steady demand rate in the infinite horizon. Our paper exhibits relative similarities to each of the reviewed papers that model the existence of an environmental regulation policy in a multi-echelon setting. However, different from the majority of the papers in this area (i.e., Benjaafar et al. 2013; Krass et al. 2013; Jaber et al. 2013; Du et al. 2011), we focus on coordination within the context of inventory replenishment decisions, and we consider a cap-and-trade and a tax policy. We propose coordination mechanisms to align each firm's objective with the supply chain's objective. Dong et al. (2014) is the only paper with a similar focus under a cap-andtrade policy, but unlike those authors, we assume that both the buyer and the vendor are subject to the policy, and our modeling allows for cases in which the purchasing price of a unit carbon allowance is greater than its selling price.

As the world economy becomes increasingly conscious of the environmental concerns, it is more likely that we will evidence complex settings where several parties in the supply chain may be subject to emission policies. In fact, Center for Climate and Energy Solutions reports California cap-and-trade program and European Union (EU) Emission Trading Scheme as examples of multi-sector cap-and-trade programs (see Center for Climate and Energy Solutions 2014). Electricity, heat and steam production, oil, iron and steel, cement, glass, pulp and paper are industries in EU's Emission Trading System, and electricity, ground transportation, heating fuels are industries in California's cap-and-trade program. This obviously indicates a need for models that analyze multiple parties in the supply chain being subject to emission policies.

Another distinguishing characteristic of our models is the difference between the purchasing and selling prices of unit carbon allowance, which leads to a piecewise objective function in both the decentralized and centralized models. Through a careful analysis of the structural properties of the objective functions, we propose finite-time exact solution procedures for these optimization problems. Our consideration of the cap-and-trade policy for both parties 
and the difference in carbon trading prices leads to some novel coordination mechanisms based on carbon credit sharing. We also extend our modeling and analysis to the case of tax policy. A final contribution of our paper is that for both policies, we investigate the impact of coordination on the environment in terms of the resulting carbon emissions. Our numerical analysis for the cap-and-trade policy and our analytical results for the tax policy show that coordination may not always be good for the environment.

In the next section, we begin with the problem definition and formulation under the two policies. In Sect. 3, we present our analytical and numerical results for the cap-and-trade policy. We then continue in Sect. 4 with an analysis for the tax policy. We conclude the paper in Sect. 5 with a discussion of our findings.

\section{Problem definition and notation}

We consider a system that consists of a buyer (retailer) and a vendor (manufacturer). The buyer and the vendor operate to meet the deterministic demand of a single product in the infinite horizon using a lot-for-lot policy. That is, the quantity produced by the manufacturer at each setup is equal to the retailer's ordering lot size. Shortages are not allowed and the replenishment lead times are zero (or, equivalently, deterministic in this setting). The vendor incurs a setup cost of $K_{v}$ monetary units at each production run, and the buyer incurs a fixed cost of $K_{b}$ monetary units at each ordering. The vendor and the buyer are subject to cost rates $h_{v}$ and $h_{b}$, respectively, for each unit held in the inventory for a unit time. It is important to note that the joint replenishment decisions in this setting have been previously studied by Banerjee and Burton (1994) and Lu (1995). In this paper, we model the carbon emissions of the buyer and the vendor resulting from production- and inventory-related activities, and we study how replenishment decisions can be coordinated under a cap-and-trade policy and a tax policy. Table 1 introduces the notation that will be used in our modeling for both policies. Without any loss of generality, the time unit is taken as a year.

In order to arrive at a coordinated solution for the two-echelon system, we study two models under each policy: the decentralized model and the centralized model. In the decentralized model, the buyer's independent replenishment decisions minimizing his/her cost per unit time determine the vendor's replenishment lot size. In the centralized model, the buyer's and vendor's costs and constraints are simultaneously taken into account to find a quantity that minimizes the total system cost per unit time. Using the centralized solution as a benchmark, we develop mechanisms that utilize price discounts, carbon credit sharing, and fixed payments to coordinate the system.

\subsection{Modeling of the different solution approaches under the cap-and-trade policy}

Under a cap-and-trade policy, both the buyer and the vendor have carbon caps (i.e., a carbon emission quota per unit time). They both emit carbon due to production/ordering setups, inventory holding, and procurement. If the emissions per unit time of one party exceed his/her cap, then he/she buys carbon credits at a rate of $p_{c}^{b}$ monetary units for one unit carbon emission. If the emissions per unit time are below the cap, then the excess amount of carbon credit is sold at a rate of $p_{c}^{s}$ monetary units for unit carbon emission $\left(p_{c}^{s} \leq p_{c}^{b}\right)$. Buying and selling carbon credits can be compared to buying and selling shares in a stock market. The difference $p_{c}^{b}-p_{c}^{s}$ can be considered as the gap between the bid and asking prices for the allowance of emitting one unit carbon. The particular values of $p_{c}^{b}$ and $p_{c}^{s}$ are determined by the supply and demand for carbon allowances in the market. Nouira et al. (2014) reports 
Table 1 Buyer's and vendor's production/inventory- and emission-related parameters

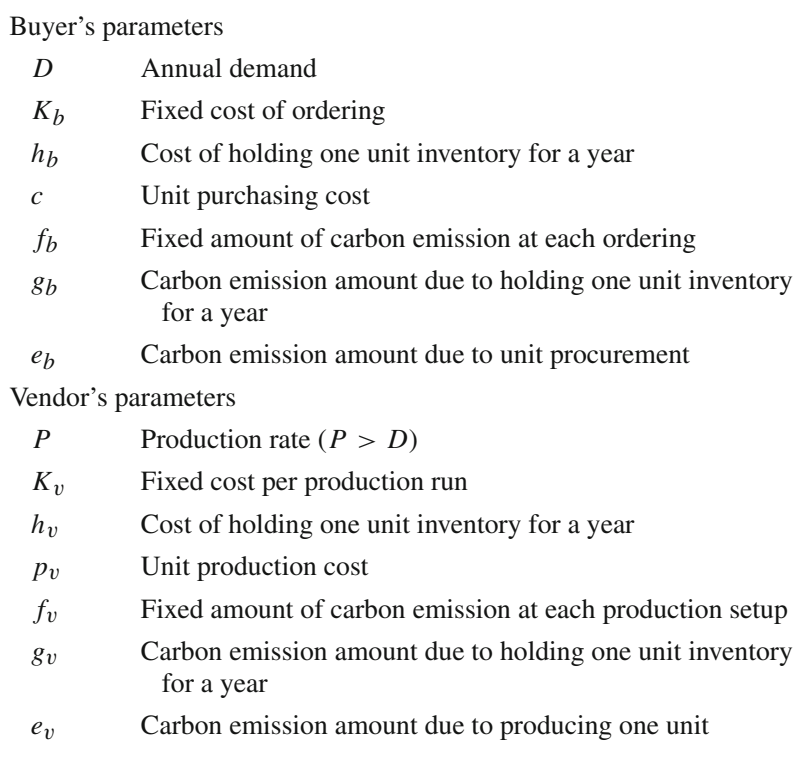

that in most cases $p_{c}^{b}>p_{c}^{s}$ due to differences in transaction costs for selling and purchasing allowances. Table 2 summarizes the additional notation specific to our discussion for the cap-and-trade policy.

Under a cap-and-trade policy, the buyer's average annual cost is given by

$$
B C\left(Q, X_{b}\right)= \begin{cases}B C_{1}\left(Q, X_{b}\right) & \text { if } X_{b} \leqslant 0 \\ B C_{2}\left(Q, X_{b}\right) & \text { if } X_{b}>0\end{cases}
$$

where

$$
B C_{1}\left(Q, X_{b}\right)=\frac{K_{b} D}{Q}+\frac{h_{b} Q}{2}+c D-p_{c}^{b} X_{b}
$$

and

$$
B C_{2}\left(Q, X_{b}\right)=\frac{K_{b} D}{Q}+\frac{h_{b} Q}{2}+c D-p_{c}^{s} X_{b} .
$$

If the buyer buys carbon credits (i.e., $X_{b}$ is negative), his/her annual cost function is given by Expression (2). If the buyer sells carbon credits (i.e., $X_{b}$ is positive), his/her annual cost function is given by Expression (3). Note that if the buyer neither sells nor buys carbon credits (i.e., $\left.X_{b}=0\right)$, then $B C_{1}\left(Q, X_{b}\right)=B C_{2}\left(Q, X_{b}\right)$.

The buyer's average annual emission when $Q$ units are ordered amounts to

$$
\frac{f_{b} D}{Q}+\frac{g_{b} Q}{2}+e_{b} D
$$

When no emission regulation policy is in place, $Q_{d}^{0}=\sqrt{\frac{2 K_{b} D}{h_{b}}}$ minimizes the buyer's annual costs and $\hat{Q}_{d}=\sqrt{\frac{2 f_{b} D}{g_{b}}}$ minimizes his/her annual emissions. 
Table 2 Problem parameters and decision variables under the cap-and-trade policy

\begin{tabular}{|c|c|}
\hline \multicolumn{2}{|l|}{ Policy parameters } \\
\hline$C_{b}$ & Buyer's annual carbon emission cap \\
\hline$C_{v}$ & Vendor's annual carbon emission cap \\
\hline$p_{c}^{b}$ & Buying price of unit carbon emission \\
\hline$p_{c}^{s}$ & Selling price of unit carbon emission \\
\hline \multicolumn{2}{|l|}{ Decision variables } \\
\hline$Q$ & Buyer's order quantity (vendor's production lot size) \\
\hline$X_{b}$ & Amount of carbon credit traded by the buyer \\
\hline$X_{v}$ & Amount of carbon credit traded by the vendor \\
\hline$X_{S}$ & $\begin{array}{l}\text { Amount of carbon credit traded by the system in the centralized model with } \\
\text { carbon credit sharing }\end{array}$ \\
\hline \multicolumn{2}{|c|}{ Functions and optimal values of decision variables } \\
\hline$B C\left(Q, X_{b}\right)$ & Buyer's average annual costs as a function of $Q$ and $X_{b}$ \\
\hline$V C\left(Q, X_{v}\right)$ & Vendor's average annual costs as a function of $Q$ and $X_{v}$ \\
\hline$T C\left(Q, X_{b}, X_{v}\right)$ & $\begin{array}{l}\text { Total average annual costs as a function of } Q, X_{b} \text { and } \\
\quad X_{v}\left(T C\left(Q, X_{b}, X_{v}\right)=B C\left(Q, X_{b}\right)+V C\left(Q, X_{v}\right)\right)\end{array}$ \\
\hline$S C\left(Q, X_{S}\right)$ & $\begin{array}{l}\text { Total average annual costs of the buyer-vendor system in the centralized } \\
\text { model with carbon credit sharing }\end{array}$ \\
\hline$Q_{d}^{*}$ & Optimal order quantity as a result of the decentralized model \\
\hline$Q_{c}^{*}$ & Optimal order quantity as a result of the centralized model \\
\hline$Q_{s}^{*}$ & $\begin{array}{l}\text { Optimal order quantity as a result of the centralized model with carbon credit } \\
\text { sharing }\end{array}$ \\
\hline
\end{tabular}

Similar to Expression (1), the vendor's annual cost is given by

$$
V C\left(Q, X_{v}\right)= \begin{cases}V C_{1}\left(Q, X_{v}\right) & \text { if } X_{v} \leqslant 0 \\ V C_{2}\left(Q, X_{v}\right) & \text { if } X_{v}>0\end{cases}
$$

where

$$
V C_{1}\left(Q, X_{v}\right)=\frac{K_{v} D}{Q}+\frac{h_{v} D Q}{2 P}+p_{v} D-p_{c}^{b} X_{v}
$$

and

$$
V C_{2}\left(Q, X_{v}\right)=\frac{K_{v} D}{Q}+\frac{h_{v} D Q}{2 P}+p_{v} D-p_{c}^{s} X_{v}
$$

If the vendor buys carbon credits (i.e., $X_{v}$ is negative), his/her annual cost can be obtained by Expression (6), and if he/she sells carbon credits (i.e., $X_{v}$ is positive), it can be obtained by Expression (7). If $X_{v}=0$, then $V C_{1}\left(Q, X_{v}\right)=V C_{2}\left(Q, X_{v}\right)$.

The vendor's average annual emission when he/she produces $Q$ units at each setup is

$$
\frac{f_{v} D}{Q}+\frac{g_{v} D Q}{2 P}+e_{v} D
$$


The decentralized model and the corresponding centralized model are then as follows:

Decentralized Model:

$$
\begin{array}{ll}
\text { Min } & B C\left(Q, X_{b}\right) \\
\text { s.t. } & \frac{f_{b} D}{Q}+\frac{g_{b} Q}{2}+e_{b} D+X_{b}=C_{b}, \\
& Q \geq 0 .
\end{array}
$$

\section{Centralized Model:}

$$
\begin{array}{ll}
\text { Min } & T C\left(Q, X_{b}, X_{v}\right) \\
\text { s.t. } & \frac{f_{b} D}{Q}+\frac{g_{b} Q}{2}+e_{b} D+X_{b}=C_{b}, \\
& \frac{f_{v} D}{Q}+\frac{g_{v} D Q}{2 P}+e_{v} D+X_{v}=C_{v}, \\
& Q \geq 0 .
\end{array}
$$

In the decentralized model presented above, the buyer only considers his/her emission constraint to minimize $B C\left(Q, X_{b}\right)$. In the centralized model, the first and the second constraints belong to the buyer and the vendor, respectively. Since these constraints have to be satisfied at any feasible solution, with a slight change of notation, we will refer to the buyer's and the vendor's traded amounts of carbon credits for replenishing $Q$ units by $X_{b}(Q)$ and $X_{v}(Q)$. Note that $X_{b}(Q)=C_{b}-\frac{f_{b} D}{Q}-\frac{g_{b} Q}{2}-e_{b} D$ and $X_{v}(Q)=C_{v}-\frac{f_{v} D}{Q}-\frac{g_{v} D Q}{2 P}-e_{v} D$. The buyer's optimal order quantity in the optimal solution of the decentralized model, $Q_{d}^{*}$, therefore, leads to $X_{b}\left(Q_{d}^{*}\right)$ and $X_{v}\left(Q_{d}^{*}\right)$ as the traded amounts of carbon credits by the buyer and the vendor. Similarly, in the optimal solution of the centralized model, the traded amounts of carbon credit by the buyer and the vendor are given by $X_{b}\left(Q_{c}^{*}\right)$ and $X_{v}\left(Q_{c}^{*}\right)$, respectively.

In order for this buyer-vendor system to achieve its maximum supply chain profitability, we propose coordination mechanisms that entail carbon credit sharing. To this end, we introduce a third model, which we refer to as the "centralized model with carbon credit sharing". In this model, it is assumed that if one party has an excess carbon allowance, he/she can make it available to the other party if that party needs it. Therefore, the average annual costs of the buyer-vendor system under carbon credit sharing are given by

$$
S C\left(Q, X_{s}\right)= \begin{cases}S C_{1}\left(Q, X_{s}\right) & \text { if } X_{s} \leqslant 0 \\ S C_{2}\left(Q, X_{S}\right) & \text { if } X_{s}>0\end{cases}
$$

where

$$
S C_{1}\left(Q, X_{s}\right)=\frac{\left(K_{b}+K_{v}\right) D}{Q}+\frac{\left(h_{b}+\frac{h_{v} D}{P}\right) Q}{2}+\left(c+p_{v}\right) D-p_{c}^{b} X_{s},
$$

and

$$
S C_{2}\left(Q, X_{s}\right)=\frac{\left(K_{b}+K_{v}\right) D}{Q}+\frac{\left(h_{b}+\frac{h_{v} D}{P}\right) Q}{2}+\left(c+p_{v}\right) D-p_{c}^{s} X_{s} .
$$

Assuming carbon credit sharing is available, the centralized model is as follows:

Centralized Model with Carbon Credit Sharing:

$$
\begin{array}{ll}
\text { Min } & S C\left(Q, X_{s}\right) \\
\text { s.t. } & \frac{\left(f_{b}+f_{v}\right) D}{Q}+\frac{\left(g_{b}+\frac{g_{v} D}{P}\right) Q}{2}+\left(e_{b}+e_{v}\right) D+X_{s}=C_{b}+C_{v} \\
& Q \geq 0 .
\end{array}
$$

Observe that, for any triplet $\left(Q, X_{b}(Q), X_{v}(Q)\right)$, there exists a feasible point $\left(Q, X_{S}(Q)\right)$ for the centralized model with carbon credit sharing, where $X_{s}(Q)=X_{b}(Q)+X_{v}(Q)$. Since $p_{c}^{b} \leq p_{c}^{s}, T C\left(Q, X_{b}(Q), X_{v}(Q)\right)$ may not be equal to $S C\left(Q, X_{s}(Q)\right)$. In fact, for any $Q \geq 0$ we have $S C\left(Q, X_{s}(Q)\right) \leq T C\left(Q, X_{b}(Q), X_{v}(Q)\right)$. More specifically, 


$$
\begin{aligned}
& T C\left(Q, X_{b}(Q), X_{v}(Q)\right)-S C\left(Q, X_{s}(Q)\right) \\
& \quad= \begin{cases}\left(p_{c}^{b}-p_{c}^{s}\right) \min \left\{-X_{b}(Q), X_{v}(Q)\right\} & \text { if } X_{b}(Q)<0 \text { and } X_{v}(Q)>0, \\
\left(p_{c}^{b}-p_{c}^{s}\right) \min \left\{X_{b}(Q),-X_{v}(Q)\right\} & \text { if } X_{b}(Q)>0 \text { and } X_{v}(Q)<0, \\
0 & \text { o.w. }\end{cases}
\end{aligned}
$$

The above expression implies that when $p_{c}^{b}>p_{c}^{s}$, there exists a difference between the total average annual costs of the two models (centralized models with or without carbon credit sharing) when one party needs to purchase carbon allowances and the other one requires fewer permits at the traded ordering lot size. If both parties need to purchase carbon allowances, or if both parties have excess allowances to sell, then there is no difference between the objective function values of the two models. It follows due to Expression (12) that we have $S C\left(Q_{s}^{*}, X_{s}\left(Q_{s}^{*}\right)\right) \leq T C\left(Q_{c}^{*}, X_{b}\left(Q_{c}^{*}\right), X_{v}\left(Q_{c}^{*}\right)\right)$ at the optimal solutions of the two models. Since carbon credit sharing has the potential to increase supply chain profitability further, we consider $S C\left(Q_{s}^{*}, X_{s}\left(Q_{s}^{*}\right)\right)$ as the least possible cost that the buyer-vendor system can achieve. Therefore, we use the solution of the centralized model with carbon credit sharing as a benchmark to propose a coordinated solution. In the next section, we start with analyzing the decentralized model and the centralized model with carbon credit sharing, and provide solution algorithms.

\subsection{Modeling of the different solution approaches under the tax policy}

An external carbon tax is applied by regulatory agencies, and a linear tax schedule is adopted. That is, the buyer and the vendor pay a monetary amount for each unit of carbon emitted. We consider a general case in which the buyer's and the vendor's tax rates are different, allowing for settings where the parties operate in different geographical locations (e.g., different countries) and/or in different industries. Table 3 summarizes the additional notation specific to our discussion for the tax policy.

Table 3 Problem parameters and decision variables under the tax policy

$\begin{array}{ll}\begin{array}{ll}\text { Policy parameters } \\ t_{b}\end{array} & \text { Carbon tax paid by the buyer for a unit emission } \\ t_{v} & \text { Carbon tax paid by the vendor for a unit emission } \\ \text { Decision variables } & \text { Buyer's order quantity (vendor's production lot size) } \\ Q & \text { Buyer's average annual costs as a function of } Q \\ \text { Functions and optimal values of decision variables } \\ B C(Q) & \text { Vendor's average annual costs as a function of } Q \\ V C(Q) & \text { Total average annual costs as a function of } Q(T C(Q)=B C(Q)+V C(Q)) \\ T C(Q) & \text { Average annual tax paid by the buyer as a function of order size } Q \\ B T(Q): & \text { Average annual tax paid by the vendor as a function of order size } Q \\ V T(Q): & \text { Average annual tax paid by the buyer-vendor system as a function of order } \\ T T(Q): & \text { size } Q(T T(Q)=B T(Q)+V T(Q)) \\ Q_{d}^{*} & \text { Optimal order quantity as a result of the decentralized model } \\ Q_{c}^{*} & \text { Optimal order quantity as a result of the centralized model }\end{array}$


In the decentralized model, the buyer solves the following replenishment problem to decide the order quantity that minimizes his/her costs:

$$
\begin{aligned}
\min B C(Q)= & \frac{\left(K_{b}+t_{b} f_{b}\right) D}{Q}+\frac{\left(h_{b}+t_{b} g_{b}\right) Q}{2}+\left(c+t_{b} e_{b}\right) D \\
Q \geq 0, &
\end{aligned}
$$

where $t_{b} f_{b}$ is the emission tax paid per replenishment, $t_{b} g_{b}$ is the emission tax paid per unit held in inventory per unit time, and $t_{b} e_{b}$ is the emission tax paid per unit ordered by the buyer. Since $B T(Q)=\frac{t_{b} f_{b} D}{Q}+\frac{t_{b} g_{b} Q}{2}+t_{b} e_{b} D$, it turns out that $B C(Q)=\frac{K_{b} D}{Q}+\frac{h_{b} Q}{2}+c D+B T(Q)$.

The vendor's average annual cost as a function of $Q$ is given by

$$
V C(Q)=\frac{\left(K_{v}+t_{v} f_{v}\right) D}{Q}+\frac{\left(h_{v}+t_{v} g_{v}\right) Q D}{2 P}+\left(p_{v}+t_{v} e_{v}\right) D,
$$

where $t_{v} f_{v}$ is the emission tax paid per production run, $t_{v} g_{v}$ is the emission tax paid per unit held in inventory per unit time, and $t_{v} e_{v}$ is the emission tax paid per unit produced by the vendor. Since $V T(Q)=\frac{t_{v} f_{v} D}{Q}+\frac{t_{v} g_{v} Q D}{2 P}+t_{v} e_{v} D$, it turns out that $V C(Q)=$ $\frac{K_{v} D}{Q}+\frac{h_{v} Q D}{2 P}+p_{v} D+V T(Q)$.

In the centralized model, the order quantity that minimizes the total cost of the system (i.e, the total cost of the buyer and the vendor) is determined. In mathematical terms, the following problem is solved.

$$
\begin{aligned}
\min T C(Q)= & \frac{\left(K_{b}+K_{v}+t_{b} f_{b}+t_{v} f_{v}\right) D}{Q}+\frac{\left[h_{b}+t_{b} g_{b}+\frac{D}{P}\left(h_{v}+t_{v} g_{v}\right)\right] Q}{2} \\
& +\left(c+p_{v}+t_{b} e_{b}+t_{v} e_{v}\right) D \\
& Q \geq 0 .
\end{aligned}
$$

\section{Analysis of the solution approaches under the cap-and-trade policy}

In this section, we provide an analysis of the decentralized model and the centralized model with carbon credit sharing to find $Q_{d}^{*}$ and $Q_{s}^{*}$. Since the objective functions in the two models exhibit piecewise forms, we propose algorithmic solutions based on some structural properties of the two problems. The proofs of all results will be presented in the "Appendix".

\subsection{Decentralized model}

As implied by Expression (1), $B C\left(Q, X_{b}\right)$ is given by either $B C_{1}\left(Q, X_{b}\right)$ or $B C_{2}\left(Q, X_{b}\right)$. In a feasible solution of the decentralized model, the buyer trades $X_{b}(Q)$ units of carbon credits. Therefore, for a feasible solution pair of $Q$ and $X_{b}(Q)$, we have

$$
B C_{1}\left(Q, X_{b}(Q)\right)=\frac{\left(K_{b}+p_{c}^{b} f_{b}\right) D}{Q}+\frac{\left(h_{b}+p_{c}^{b} g_{b}\right) Q}{2}+\left(c+p_{c}^{b} e_{b}\right) D-p_{c}^{b} C_{b}
$$

Note that $B C_{1}\left(Q, X_{b}(Q)\right)$ is a strictly convex function of $Q$ with a unique minimizer at

$$
Q_{d 1}^{*}=\sqrt{\frac{2\left(K_{b}+p_{c}^{b} f_{b}\right) D}{h_{b}+p_{c}^{b} g_{b}}} .
$$


Likewise, for a feasible solution pair of $Q$ and $X_{b}(Q), B C_{2}\left(Q, X_{b}(Q)\right)$ can be rewritten as

$$
B C_{2}\left(Q, X_{b}(Q)\right)=\frac{\left(K_{b}+p_{c}^{s} f_{b}\right) D}{Q}+\frac{\left(h_{b}+p_{c}^{s} g_{b}\right) Q}{2}+\left(c+p_{c}^{s} e_{b}\right) D-p_{c}^{s} C_{b} .
$$

$B C_{2}\left(Q, X_{b}(Q)\right)$ is also a strictly convex function with a unique minimizer at

$$
Q_{d 2}^{*}=\sqrt{\frac{2\left(K_{b}+p_{c}^{s} f_{b}\right) D}{h_{b}+p_{c}^{s} g_{b}}} .
$$

Lemma 1 If $\left(C_{b}-e_{b} D\right) \leq \sqrt{2 g_{b} f_{b} D}$, then the buyer does not sell carbon credits at any order quantity, that is $X_{b}(Q) \leq 0$ for all $Q$, and $Q_{d}^{*}=Q_{d 1}^{*}$.

Lemma 1 and its proof imply that if the annual cap is smaller than even the minimum annual emission possible by ordering decisions, then regardless of what quantity is ordered, the buyer has to purchase carbon credits. As discussed in Sect. 2, when $X_{b}(Q)=0$, the buyer neither purchases nor sells carbon credits. If $\left(C_{b}-e_{b} D\right)^{2} \geq 2 g_{b} f_{b} D$, there are two order quantities, which we refer to as $Q_{1}$ and $Q_{2}$, satisfying $X_{b}(Q)=0$. In terms of the problem parameters, these quantities are given by

$$
Q_{1}=\frac{C_{b}-e_{b} D-\sqrt{\left(C_{b}-e_{b} D\right)^{2}-2 g_{b} f_{b} D}}{g_{b}}
$$

and

$$
Q_{2}=\frac{C_{b}-e_{b} D+\sqrt{\left(C_{b}-e_{b} D\right)^{2}-2 g_{b} f_{b} D}}{g_{b}} .
$$

If $\left(C_{b}-e_{b} D\right)^{2}>2 g_{b} f_{b} D$, we take $Q_{2}$ as the larger root, i.e., $Q_{2}>Q_{1}$.

The results in the seven lemmas (Lemmas 2-8) and the two corollaries (Corollaries 4 and 5) presented in the "Appendix" lead us to the different possible solutions that can happen in case of $\left(C_{b}-e_{b} D\right)>\sqrt{2 g_{b} f_{b} D}$. These results, jointly with Lemma 1, yield the optimal solution algorithm, Algorithm 1. Based on Lemmas 2-8 and Corollaries 4-5 we establish the fact that the ordinal relation between $f_{b} h_{b}$ and $K_{b} g_{b}$ is important. Specifically, we show step by step that if $f_{b} h_{b}=K_{b} g_{b}$, then $Q_{d}^{*}=Q_{d 2}^{*}$, and the optimal solution in the other cases (i.e., $f_{b} h_{b}<K_{b} g_{b}$ and $f_{b} h_{b}>K_{b} g_{b}$ ) depends on the ordering among $Q_{1}, Q_{2}, Q_{d 1}^{*}$, and $Q_{d 2}^{*}$. We present Algorithm 1 next.

\section{Algorithm 1: Solution of the Decentralized Model}

1. If $\left(C_{b}-e_{b} D\right) \leq \sqrt{2 g_{b} f_{b} D}$, then set $Q_{d}^{*}=Q_{d 1}^{*}$.

2. If $\left(C_{b}-e_{b} D\right)>\sqrt{2 g_{b} f_{b} D}$, then do the following:

(a) If $f_{b} h_{b}=K_{b} g_{b}$, set $Q_{d}^{*}=Q_{d 2}^{*}$.

(b) If $f_{b} h_{b}<K_{b} g_{b}$, and

i. if $Q_{2} \leq Q_{d 1}^{*}$, set $Q_{d}^{*}=Q_{d 1}^{*}$,

ii. else,

A. if $Q_{2} \geq Q_{d 2}^{*}$, set $Q_{d}^{*}=Q_{d 2}^{*}$,

B. if $Q_{2}<Q_{d 2}^{*}$, set $Q_{d}^{*}=Q_{2}$.

(c) If $f_{b} h_{b}>K_{b} g_{b}$, and

i. if $Q_{d 1}^{*} \leq Q_{1}$, set $Q_{d}^{*}=Q_{d 1}^{*}$,

ii. else,
A. if $Q_{d 2}^{*} \geq Q_{1}$, set $Q_{d}^{*}=Q_{d 2}^{*}$,
B. if $Q_{d 2}^{*}<Q_{1}$, set $Q_{d}^{*}=Q_{1}$. 
Theorem 1 Algorithm 1 gives the optimal solution to the retailer's replenishment problem formulated in the decentralized model.

Recall from Corollary 4 the three possible orderings among $Q_{1}, Q_{2}, Q_{d 1}^{*}$, and $Q_{d 2}^{*}$ in the case of $\left(C_{b}-e_{b} D\right)>\sqrt{2 g_{b} f_{b} D}$ and $f_{b} h_{b}<K_{b} g_{b}$. Theorem 1 and its proof imply that if $Q_{1}<Q_{2} \leq Q_{d 1}^{*}<Q_{d 2}^{*}$, then $Q_{d}^{*}=Q_{d 1}^{*}$; if $Q_{1}<Q_{d 1}^{*}<Q_{d 2}^{*} \leq Q_{2}$, then $Q_{d}^{*}=Q_{d 2}^{*}$; if $Q_{1}<Q_{d 1}^{*}<Q_{2}<Q_{d 2}^{*}$, then $Q_{d}^{*}=Q_{2}$. Similarly, in the case of $\left(C_{b}-e_{b} D\right)>\sqrt{2 g_{b} f_{b} D}$ and $f_{b} h_{b}>K_{b} g_{b}$, there are three possible orderings among $Q_{1}, Q_{2}, Q_{d 1}^{*}$, and $Q_{d 2}^{*}$, as stated in Corollary 5. If $Q_{1} \leq Q_{d 2}^{*}<Q_{d 1}^{*}<Q_{2}$, then $Q_{d}^{*}=Q_{d 2}^{*}$; if $Q_{d 2}^{*}<Q_{1}<Q_{d 1}^{*}<Q_{2}$, then $Q_{d}^{*}=Q_{1}$; if $Q_{d 2}^{*}<Q_{d 1}^{*} \leq Q_{1}<Q_{2}$, then $Q_{d}^{*}=Q_{d 1}^{*}$. Theorem 1 has a further implication in terms of the sensitivity of the optimal order quantity to changes in $C_{b}$. We present this result in the next corollary.

\section{Corollary 1 Let us assume that the cap is increased above its current value $C_{b}$.}

- If $f_{b} h_{b}=K_{b} g_{b}$, then optimal order quantity $Q_{d}^{*}$ does not change, and its value is given by $Q_{d 1}^{*}$.

- If $f_{b} h_{b}<K_{b} g_{b}, Q_{d}^{*}$ either stays the same or increases (i.e., $Q_{d}^{*}$ is nondecreasing in $C_{b}$ ).

- If $f_{b} h_{b}>K_{b} g_{b}, Q_{d}^{*}$ either stays the same or decreases (i.e., $Q_{d}^{*}$ is nonincreasing in $C_{b}$ ).

The above corollary is presented without a proof. However, a formal proof would be based on Lemma 1, Lemma 5, Corollary 4, Corollary 5, Theorem 1, and the fact that $Q_{2}$ is increasing in $C_{b}$ and $Q_{1}$ is decreasing in $C_{b}$. Let us define $Q_{1}^{\prime}$ and $Q_{2}^{\prime}$ as the two quantities that satisfy $X_{b}(Q)=0$ under the increased value of $C_{b}$. We have $Q_{1}^{\prime}<Q_{1}$ and $Q_{2}^{\prime}>Q_{2}$. For example, in an instance of the problem where $\left(C_{b}-e_{b} D\right)>\sqrt{2 g_{b} f_{b} D}$ and $f_{b} h_{b}<K_{b} g_{b}$, if $Q_{1}<Q_{d 1}^{*}<Q_{2}<Q_{d 2}^{*}$ at the current value of $C_{b}$, Corollary 4 implies that $Q_{d}^{*}=Q_{2}$ and either one of the following two orderings happens if $C_{b}$ is increased: $Q_{1}^{\prime}<Q_{d 1}^{*}<Q_{2}^{\prime}<Q_{d 2}^{*}$ or $Q_{1}^{\prime}<Q_{d 1}^{*}<Q_{d 2}^{*} \leq Q_{2}^{\prime}$. In the former case, the new optimal order quantity is $Q_{2}^{\prime}$, which is greater than $Q_{2}$. In the latter case, the new optimal order quantity is $Q_{d 2}^{*}$, which again is greater than $Q_{2}$. Following a similar reasoning for each possible case of the problem leads to Corollary 1.

Corollary 1 is significant for a policy maker to foresee what kind of an effect a change in $C_{b}$ will have on the quantity traded at each dispatch. It also suggests that knowing how the ratio of fixed ordering cost to inventory holding cost rate (i.e., $\frac{K_{b}}{h_{b}}$ ) compares to the ratio of fixed carbon emission amount at each ordering to carbon emission rate due to inventory holding (i.e., $\frac{f_{b}}{g_{b}}$ ) is sufficient for this prediction. For example, if $\frac{K_{b}}{h_{b}}<\frac{f_{b}}{g_{b}}$, increasing the cap may result in a fall in the quantity traded at each dispatch.

Next, we proceed with a similar analysis for the centralized model with carbon credit sharing.

\subsection{Centralized model with carbon credit sharing}

In a feasible solution of the centralized model with carbon credit sharing, the system trades $X_{S}(Q)$ units of carbon credits, where $X_{S}(Q)=C_{b}+C_{v}-\frac{\left(f_{b}+f_{v}\right) D}{Q}-\frac{\left(g_{b}+\frac{g_{v} D}{P}\right) Q}{2}-\left(e_{b}+e_{v}\right) D$. For this pair of order quantity and traded amount of carbon credits, it turns out that

$$
\begin{aligned}
S C_{1}\left(Q, X_{s}(Q)\right)= & \frac{\left(K_{b}+K_{v}+p_{c}^{b}\left(f_{b}+f_{v}\right)\right) D}{Q}+\frac{\left(h_{b}+\frac{h_{v} D}{Q}+p_{c}^{b}\left(g_{b}+\frac{g_{v} D}{P}\right)\right) Q}{2} \\
& +\left(c+p_{v}+p_{c}^{b}\left(e_{b}+e_{v}\right)\right) D-p_{c}^{b}\left(C_{b}+C_{v}\right) .
\end{aligned}
$$


The above expression is strictly convex in $Q$ with a unique minimizer at

$$
Q_{c 1}^{*}=\sqrt{\frac{2\left(K_{b}+K_{v}+p_{c}^{b}\left(f_{b}+f_{v}\right)\right) D}{h_{b}+\frac{h_{v} D}{P}+p_{c}^{b}\left(g_{b}+\frac{g_{v} D}{P}\right)}} .
$$

A similar expression can be derived for $S C_{2}\left(Q, X_{S}(Q)\right)$ and is given by

$$
\begin{aligned}
S C_{2}\left(Q, X_{s}(Q)\right)= & \frac{\left(K_{b}+K_{v}+p_{c}^{s}\left(f_{b}+f_{v}\right)\right) D}{Q}+\frac{\left(h_{b}+\frac{h_{v} D}{Q}+p_{c}^{s}\left(g_{b}+\frac{g_{v} D}{P}\right)\right) Q}{2} \\
& +\left(c+p_{v}+p_{c}^{s}\left(e_{b}+e_{v}\right)\right) D-p_{c}^{s}\left(C_{b}+C_{v}\right) .
\end{aligned}
$$

$S C_{2}\left(Q, X_{b}(Q)\right)$ is also a strictly convex function with a unique minimizer at

$$
Q_{c 2}^{*}=\sqrt{\frac{2\left(K_{b}+K_{v}+p_{c}^{s}\left(f_{b}+f_{v}\right)\right) D}{h_{b}+\frac{h_{v} D}{P}+p_{c}^{s}\left(g_{b}+\frac{g_{v} D}{P}\right)}} .
$$

Expression (9) is similar to Expression (1) in its structural properties. Therefore, results similar to those proved in Sect. 3.1 for the decentralized model also hold for the centralized model with carbon sharing. If $\left[C_{b}+C_{v}-\left(e_{b}+e_{v}\right) D\right] \leq \sqrt{2\left(g_{b}+\frac{g_{v} D}{P}\right)\left(f_{b}+f_{v}\right) D}$, then the buyer-vendor system does not sell carbon credits at any order quantity, that is $X_{S}(Q) \leq 0$ for all $Q$. When $\left[C_{b}+C_{v}-\left(e_{b}+e_{v}\right) D\right] \geq \sqrt{2\left(g_{b}+\frac{g_{v} D}{P}\right)\left(f_{b}+f_{v}\right) D}$, we have $X_{s}(Q)=0$ at the following two values of the order quantity:

$$
Q_{3}=\frac{C_{b}+C_{v}-\left(e_{b}+e_{v}\right) D-\sqrt{\left[C_{b}+C_{v}-\left(e_{b}+e_{v}\right) D\right]^{2}-2\left(g_{b}+\frac{g_{v} D}{P}\right)\left(f_{b}+f_{v}\right) D}}{g_{b}+\frac{g_{v} D}{P}}
$$

and

$$
Q_{4}=\frac{C_{b}+C_{v}-\left(e_{b}+e_{v}\right) D+\sqrt{\left[C_{b}+C_{v}-\left(e_{b}+e_{v}\right) D\right]^{2}-2\left(g_{b}+\frac{g_{v} D}{P}\right)\left(f_{b}+f_{v}\right) D}}{g_{b}+\frac{g_{v} D}{P}} .
$$

It turns out that the system sells carbon credits only when $\left[C_{b}+C_{v}-\left(e_{b}+e_{v}\right) D\right]>$ $\sqrt{2\left(g_{b}+\frac{g_{v} D}{P}\right)\left(f_{b}+f_{v}\right) D}$ and $Q_{3}<Q<Q_{4}$.

We propose the following algorithm to obtain the optimal solution of the centralized model with carbon credit sharing. A detailed proof will not be presented because it follows the same lines as Theorem 1's proof and makes use of similar results (i.e., Lemma 1, Lemma 5, Corollary 4, and Corollary 5) that set a foundation for Theorem 1.

\section{Algorithm 2: Solution of the Centralized Model with Carbon Credit Sharing}

1. If $\left[C_{b}+C_{v}-\left(e_{b}+e_{v}\right) D\right] \leq \sqrt{2\left(g_{b}+\frac{g_{v} D}{P}\right)\left(f_{b}+f_{v}\right) D}$, then set $Q_{s}^{*}=Q_{c 1}^{*}$.

2. If $\left[C_{b}+C_{v}-\left(e_{b}+e_{v}\right) D\right]>\sqrt{2\left(g_{b}+\frac{g_{v} D}{P}\right)\left(f_{b}+f_{v}\right) D}$, then do the following:

(a) If $\left(f_{b}+f_{v}\right)\left(h_{b}+\frac{h_{v} D}{P}\right)=\left(K_{b}+K_{v}\right)\left(g_{b}+\frac{g_{v} D}{P}\right)$, set $Q_{s}^{*}=Q_{c 2}^{*}$. 
(b) If $\left(f_{b}+f_{v}\right)\left(h_{b}+\frac{h_{v} D}{P}\right)<\left(K_{b}+K_{v}\right)\left(g_{b}+\frac{g_{v} D}{P}\right)$, and

i. if $Q_{4} \leq Q_{c 1}^{*}$, set $Q_{s}^{*}=Q_{c 1}^{*}$,

ii. else,

A. if $Q_{4} \geq Q_{c 2}^{*}$, set $Q_{s}^{*}=Q_{c 2}^{*}$,

B. if $Q_{4}<Q_{c 2}^{*}$, set $Q_{s}^{*}=Q_{4}$.

(c) If $\left(f_{b}+f_{v}\right)\left(h_{b}+\frac{h_{v} D}{P}\right)>\left(K_{b}+K_{v}\right)\left(g_{b}+\frac{g_{v} D}{P}\right)$, and

(i.) if $Q_{c 1}^{*} \leq Q_{3}$, set $Q_{s}^{*}=Q_{c 1}^{*}$,

(ii.) else,
A. if $Q_{c 2}^{*} \geq Q_{3}$, set $Q_{s}^{*}=Q_{c 2}^{*}$,
B. if $Q_{c 2}^{*}<Q_{3}$, set $Q_{s}^{*}=Q_{3}$.

\subsection{Coordination mechanisms}

In this section, we present coordination mechanisms that help the buyer-vendor system to arrive at the system optimal solution by making the most efficient use of carbon credits. These coordination mechanisms assume that vendor has full information about the ordering behavior of the buyer, and the buyer orders from the current vendor as long as his/her costs as a result of the coordinated solution are not more than those under the decentralized solution. The novelty of the proposed coordination mechanisms is that they make use of carbon credit sharing. Recall that in this setting, the purchasing price of one unit carbon credit is greater than or equal to its selling price (i.e., $p_{c}^{b} \geq p_{c}^{s}$ ). In settings where $p_{c}^{b}>p_{c}^{s}$, and one party is selling carbon credits while the other party is purchasing them, the system is actually losing an opportunity to profit more due to the monetary value that the purchasing party pays to intermediary agencies (i.e., $p_{c}^{b}-p_{c}^{s}$ per unit carbon credit purchased). The lost opportunity is quantified in Expression (12). Therefore, the proposed coordination mechanisms, as part of sharing the extra benefits of the centralized solutions, entail the party who has extra carbon credits to pass them to the other party, who would otherwise purchase them at a higher price in the market. This way, we minimize the system's need to purchase carbon credits, and hence, to pay intermediary agencies.

While carbon credit sharing may lead to reduced overall costs, it may increase or decrease the total annual emissions in comparison to a coordinated solution that does not allow carbon credit sharing. The examples in Table 4 are illustrative of these two cases.

In Examples 7 and 8, carbon credit sharing reduces total average annual costs. In Example 7 , the optimal order quantity of the centralized model without carbon credit sharing $\left(Q_{c}^{*}\right)$ is 235.5 , and this quantity leads to 705.8425 as the total average annual emissions. The optimal order quantity of the centralized model with carbon credit sharing $\left(Q_{s}^{*}\right)$ is 251.5 , which results in a value of 708.145 as the total average annual emissions. While Example 7 is illustrative of a case in which carbon credit sharing increases the emissions of the buyervendor system, Example 8 exemplifies a complementary case. Specifically, in Example 8, the total average annual emissions under the optimal solution of the centralized model without carbon credit sharing is 721.987 , which reduces to 715.322 due to carbon credit sharing. These two examples show that the impact of carbon credit sharing on the environment is dependent on the specific setting; however, the total costs either stay the same or reduce due to carbon credit sharing (i.e., $S C\left(Q_{s}^{*}, X_{s}\left(Q_{s}^{*}\right)\right) \leq T C\left(Q_{c}^{*}, X_{b}\left(Q_{c}^{*}\right), X_{v}\left(Q_{c}^{*}\right)\right)$ ). Therefore, in the proposed coordination mechanisms, $S C\left(Q_{s}^{*}, X_{S}\left(Q_{s}^{*}\right)\right)$ will be considered as the minimum system costs that can be achieved. Before we introduce these coordination mechanisms, we present the following result, which is crucial for understanding why these mechanisms work. 


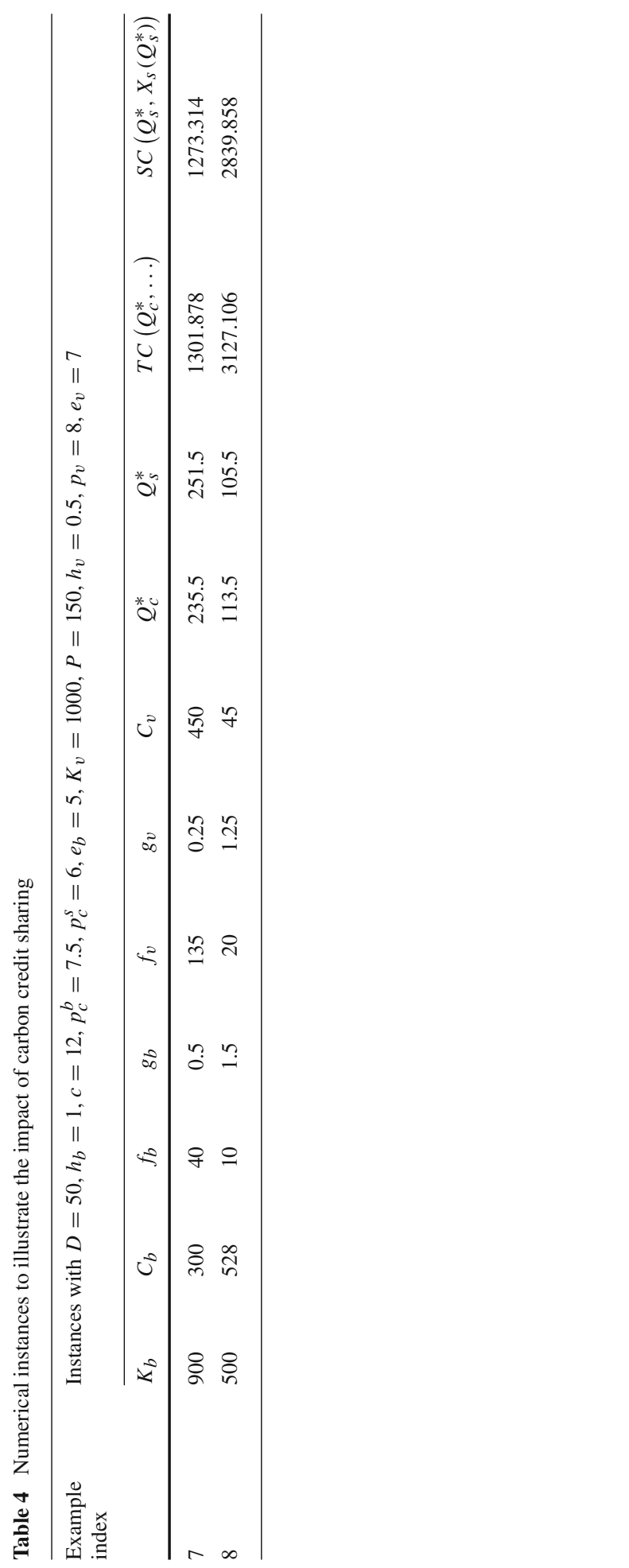


Proposition $1 B C\left(Q, X_{b}(Q)\right)$ is a strictly convex function of $Q$.

Observe that if both parties would not sell carbon credits under the system optimal quantity $Q_{s}^{*}$ (i.e., $X_{b}\left(Q_{s}^{*}\right) \leq 0$ and $X_{v}\left(Q_{s}^{*}\right) \leq 0$ ), or if both parties would not purchase carbon credits under the system optimal quantity $Q_{s}^{*}$ (i.e., $X_{b}\left(Q_{s}^{*}\right) \geq 0$ and $X_{v}\left(Q_{s}^{*}\right) \geq 0$ ), then carbon credit sharing would not bring any benefit to the system. Therefore, in those cases we rely on traditional coordination mechanisms. In fact, an implication of Proposition 1 is that quantity discounts with economies or diseconomies of scale coordinates the buyer-vendor system. Specifically, if $Q_{s}^{*}>Q_{d}^{*}$, then under a per unit discount of $d=\frac{B C\left(Q_{s}^{*}, X_{b}\left(Q_{s}^{*}\right)\right)-B C\left(Q_{d}^{*}, X_{b}\left(Q_{d}^{*}\right)\right)}{D}$ for order quantities greater than or equal to $Q_{s}^{*}$, the buyer would be indifferent to whether $Q_{d}^{*}$ or $Q_{s}^{*}$ were ordered. If $Q_{s}^{*}<Q_{d}^{*}$, under a per unit discount of the same amount for order quantities less than or equal to $Q_{s}^{*}$, the buyer would be indifferent to whether $Q_{d}^{*}$ or $Q_{s}^{*}$ were ordered.

In cases where one party would sell carbon credits while the other party would buy carbon credits under the centralized optimum quantity, we propose the following coordination mechanisms:

CM1: If $X_{b}\left(Q_{s}^{*}\right) \leq 0, X_{v}\left(Q_{s}^{*}\right) \geq 0, p_{c}^{b} \times \min \left\{-X_{b}\left(Q_{s}^{*}\right), X_{v}\left(Q_{s}^{*}\right)\right\} \geq B C\left(Q_{s}^{*}, X_{b}\left(Q_{s}^{*}\right)\right)-$ $B C\left(Q_{d}^{*}, X_{b}\left(Q_{d}^{*}\right)\right)$, and

- if $Q_{d}^{*}<Q_{s}^{*}$, then for order quantities greater than or equal to $Q_{s}^{*}$,

- if $Q_{d}^{*}>Q_{s}^{*}$, then for order quantities less than or equal to $Q_{s}^{*}$,

the vendor gives $Y=\min \left\{-X_{b}\left(Q_{s}^{*}\right), X_{v}\left(Q_{s}^{*}\right)\right\}$ carbon credits for free to the buyer and the buyer makes a fixed payment of $B C\left(Q_{d}^{*}, X_{b}\left(Q_{d}^{*}\right)\right)+p_{c}^{b} \times Y-B C\left(Q_{s}^{*}, X_{b}\left(Q_{s}^{*}\right)\right)$ to the vendor.

CM2: If $X_{b}\left(Q_{s}^{*}\right) \leq 0, X_{v}\left(Q_{s}^{*}\right) \geq 0, p_{c}^{b} \times \min \left\{-X_{b}\left(Q_{s}^{*}\right), X_{v}\left(Q_{s}^{*}\right)\right\}<B C\left(Q_{s}^{*}, X_{b}\left(Q_{s}^{*}\right)\right)-$ $B C\left(Q_{d}^{*}, X_{b}\left(Q_{d}^{*}\right)\right)$, and

- if $Q_{d}^{*}<Q_{s}^{*}$, then for order quantities greater than or equal to $Q_{s}^{*}$,

- if $Q_{d}^{*}>Q_{s}^{*}$, then for order quantities less than or equal to $Q_{s}^{*}$,

the vendor gives $Y=\min \left\{-X_{b}\left(Q_{s}^{*}\right), X_{v}\left(Q_{s}^{*}\right)\right\}$ carbon credits for free to the buyer and a per unit discount of $d=\left[B C\left(Q_{s}^{*}, X_{b}\left(Q_{s}^{*}\right)\right)-B C\left(Q_{d}^{*}, X_{b}\left(Q_{d}^{*}\right)\right)-p_{c}^{b} \times Y\right] / D$ for all items in the lot.

CM3: If $X_{b}\left(Q_{s}^{*}\right) \geq 0, X_{v}\left(Q_{s}^{*}\right) \leq 0$, and

- if $Q_{d}^{*}<Q_{s}^{*}$, then for order quantities greater than or equal to $Q_{s}^{*}$,

- if $Q_{d}^{*}>Q_{s}^{*}$, then for order quantities less than or equal to $Q_{s}^{*}$,

the buyer gives $Y=\min \left\{X_{b}\left(Q_{s}^{*}\right),-X_{v}\left(Q_{s}^{*}\right)\right\}$ carbon credits for free to the vendor and the vendor gives a per unit discount of $d=\left[B C\left(Q_{s}^{*}, X_{b}\left(Q_{s}^{*}\right)\right)-B C\left(Q_{d}^{*}, X_{b}\left(Q_{d}^{*}\right)\right)+p_{c}^{s} \times Y\right] / D$ to the buyer for all items in the lot.

The first and the second coordination mechanisms (i.e., CM1 and CM2) apply to cases in which the buyer would buy carbon credits while the vendor would sell carbon credits under the centralized optimum solution. The expression $\min \left\{-X_{b}\left(Q_{s}^{*}\right), X_{v}\left(Q_{s}^{*}\right)\right\}$ refers to the amount of carbon credits the vendor can provide to the buyer. CM1 and CM2 differ in whether the monetary value of this amount in the market (i.e., $\left.p_{c}^{b} \times \min \left\{-X_{b}\left(Q_{s}^{*}\right), X_{v}\left(Q_{s}^{*}\right)\right\}\right)$ is greater or less than the buyer's loss from using the centralized solution (i.e., $B C\left(Q_{s}^{*}, X_{b}\left(Q_{s}^{*}\right)\right)-$ $\left.B C\left(Q_{d}^{*}, X_{b}\left(Q_{d}^{*}\right)\right)\right)$. In cases where the value of carbon credits given by the vendor to the buyer exceeds the buyer's loss, then CM1 applies, and the buyer returns the extra value of 
Table 5 Numerical instances to illustrate the proposed coordination mechanisms

\begin{tabular}{|c|c|c|c|c|c|c|c|c|c|c|c|c|c|c|}
\hline \multirow{2}{*}{$\begin{array}{l}\text { Example } \\
\text { index }\end{array}$} & \multicolumn{14}{|c|}{ Instances with $D=50, c=12, p_{v}=8$} \\
\hline & $K_{b}$ & $h_{b}$ & $p_{c}^{b}$ & $C_{b}$ & $f_{b}$ & $g_{b}$ & $e_{b}$ & $K_{v}$ & $P$ & $h_{v}$ & $f_{v}$ & $g_{v}$ & $e_{v}$ & $C_{v}$ \\
\hline 9 & 900 & 1 & 7.5 & 300 & 40 & 0.5 & 5 & 1000 & 150 & 0.5 & 135 & 0.25 & 7 & 450 \\
\hline 10 & 90 & 2 & 7.5 & 345 & 90 & 1 & 5 & 1000 & 75 & 0.8 & 60 & 1.75 & 6 & 400 \\
\hline 11 & 330 & 3.2 & 2.5 & 300 & 90 & 0.5 & 4.5 & 100 & 55 & 3 & 95 & 0.25 & 6 & 350 \\
\hline
\end{tabular}

Table 6 Solutions of instances in Table 5

\begin{tabular}{|c|c|c|c|c|c|}
\hline $\begin{array}{l}\text { Example } \\
\text { index }\end{array}$ & $Q_{d}^{*}$ & $Q_{S}^{*}$ & $X_{b}\left(Q_{s}^{*}\right)$ & $X_{v}\left(Q_{S}^{*}\right)$ & Coordination mechanism \\
\hline 9 & 158.944 & 251.425 & -20.811 & 62.677 & $\begin{array}{l}\text { For } Q \geq 251.425, \text { the vendor gives } \\
20.811 \text { carbon credits to the buyer, } \\
\text { who in return, makes a fixed } \\
\text { payment of } 75.291\end{array}$ \\
\hline 10 & 89.737 & 113.186 & -1.351 & 7.470 & $\begin{array}{c}\text { For } Q \geq 113.186, \text { the vendor gives } \\
1.351 \text { carbon credits to the buyer } \\
\text { and a per unit discount of } 0.259\end{array}$ \\
\hline 11 & 110.195 & 107.345 & 6.243 & -6.448 & $\begin{array}{l}\text { For } Q \leq 107.345 \text {, buyer gives } 6.243 \\
\text { carbon credits to the vendor, who in } \\
\text { return, gives a per unit discount of } 0.253\end{array}$ \\
\hline
\end{tabular}

carbon credits as a fixed payment to the vendor. If it is less than the buyer's loss, then CM2 applies, and the vendor further gives an all-units discount to the buyer to compensate his/her remaining loss. CM3 applies to cases in which the vendor would buy carbon credits while the buyer would sell carbon credits under the centralized optimum solution. In this case, the buyer gives the vendor the carbon credits he/she needs, but in return receives a higher amount of per unit discount. The per unit discount amount is such that it compensates the buyer for his/her losses if he/she orders the centralized optimum quantity in addition to the monetary value of carbon credits he/she agrees to give to the vendor.

In Table 5, parameters of three instances are presented. In Table 6, a summary of their decentralized and centralized solutions as well as the coordinating mechanisms are reported. Examples 9, 10, and 11 are illustrative of CM1, CM2, and CM3, respectively.

\subsection{The impact of coordination on the environment under the cap-and-trade policy}

To understand the impact of coordination on the environment, we numerically compare the average annual emissions resulting from the decentralized model and the centralized model with carbon credit sharing. For this purpose, the following additional pieces of notation are used.

$T E(Q)$ : Average annual emissions of the system if order size is $Q$ units

$R$ : Ratio of average annual system emissions resulting from the two models

The ratio $R$ is a performance measure on the system's environmental quality under the centralized model with carbon credit sharing compared to its environmental performance under the decentralized model. In mathematical terms, 
Table 7 Numerical instances to illustrate the impact of coordination on emissions

\begin{tabular}{lrrrrrrrrr}
\hline $\begin{array}{l}\text { Example } \\
\text { index }\end{array}$ & \multicolumn{8}{c}{$\begin{array}{l}\text { Instances with } D=30, p_{c}^{s}=1.5, e_{b}=1, P=50, h_{v}=1.2, K_{b}=40, f_{b}=20, \\
\end{array}$} & \multicolumn{1}{c}{$g_{b}=0.5, e_{v}=1.5, C_{v}=200$} \\
\cline { 2 - 10 } & \multicolumn{1}{c}{$K_{v}$} & \multicolumn{1}{c}{$C_{b}$} & \multicolumn{1}{c}{$f_{v}$} & $g_{v}$ & $h_{b}$ & $p_{c}^{b}$ & $Q_{d}^{*}$ & $Q_{s}^{*}$ & $R$ \\
\hline 12 & 500 & 80 & 120 & 0.35 & 1.5 & 2.5 & 43.205 & 117.041 & 0.813 \\
13 & 500 & 80 & 1800 & 0.35 & 1.5 & 2.5 & 43.205 & 276.488 & 0.274 \\
14 & 8000 & 80 & 120 & 12 & 1.5 & 2.5 & 43.205 & 153.123 & 2.044 \\
15 & 500 & 80 & 120 & 0.35 & 10 & 2.5 & 19.766 & 61.793 & 0.560 \\
16 & 500 & 80 & 120 & 0.35 & 10 & 3.5 & 19.766 & 61.793 & 0.560 \\
17 & 500 & 40 & 120 & 0.35 & 1.5 & 2.5 & 44.313 & 117.041 & 0.822 \\
18 & 500 & 120 & 120 & 0.35 & 1.5 & 2.5 & 43.205 & 117.041 & 0.813 \\
\hline
\end{tabular}

$$
R=\frac{T E\left(Q_{s}^{*}\right)}{T E\left(Q_{d}^{*}\right)}=\frac{\frac{\left(f_{b}+f_{v}\right) D}{Q_{s}^{*}}+\frac{\left(g_{b}+\frac{g_{v} D}{P}\right) Q_{s}^{*}}{2}+\left(e_{b}+e_{v}\right) D}{\frac{\left(f_{b}+f_{v}\right) D}{Q_{d}^{*}}+\frac{\left(g_{b}+\frac{g_{v} D}{P}\right) Q_{d}^{*}}{2}+\left(e_{b}+e_{v}\right) D} .
$$

A value of $R>1$ would be due to $T E\left(Q_{s}^{*}\right)>T E\left(Q_{d}^{*}\right)$, implying that the coordinated solution is not good for the environment. Similarly, a value of $R<1$ implies that coordination is better for the environment than the uncoordinated solution. In Table 7, we present some instances of the problem to illustrate possible values of $R$. We would like to note that we have studied the effect of each parameter on $R$ over an extensive numerical analysis; however, there are so many interactions between the problem parameters that there is no generalizable result regarding how $R$ changes with respect to varying values of a certain parameter.

Example 12 in Table 7 can be considered as the base instance around which other examples are generated. Example 13 illustrates an instance in which $R$ is very small (i.e., 0.274) and Example 14 illustrates an instance in which $R$ is very large (i.e., 2.044). In our experimentation, we have identified that most of the instances for which $R$ is very large have extremely high $K_{v}$ values. Because, when $K_{v}$ is extremely high, the manufacturer wants to make less frequent setups and produce in larger quantities to save from average annual setup costs, and this results in higher average annual carbon emissions in the centralized model with carbon credit sharing. However, we would like to note that we have also observed instances without extremely high $K_{v}$ values to also have $R>1$. In Examples 15 and 16, the inventory holding cost rate of the buyer is so large that in both the decentralized and centralized models, it is only economically appealing to order in small lot sizes and more frequently. It turns out that in both instances, the buyer in the decentralized solution and the system in the centralized solution sell carbon credits. Therefore, even if the buying price of a unit carbon emission is different among these two instances, it does not have an effect on the optimum solutions. Examples 17 and 18 are different than the base instance in the buyer's average annual carbon emission cap, $C_{b}$. In Example 17, the buyer's annual cap is so small that he/she has to buy carbon allowances in the decentralized solution. In Example 12, on the other hand, the buyer has extra allowances to sell. Therefore, even if the buyer's annual cap is increased further in Example 18, it does not have an effect on the solution (i.e., the optimal solutions are the same as in the base instance). 


\section{Analysis of the solution approaches under the tax policy}

In this section, we provide an analysis of the decentralized model and the centralized model under the carbon tax mechanism to find the cost-minimizing order quantities $Q_{d}^{*}$ and $Q_{c}^{*}$, respectively. We also present some properties related to $Q_{d}^{*}, Q_{c}^{*}$ and average annual tax amounts of the buyer and the vendor.

The order quantity that minimizes the average annual taxes of the buyer is given by

$$
Q_{d}^{t}=\sqrt{\frac{2 f_{b} D}{g_{b}}} .
$$

As the average annual taxes are linearly proportional to the average annual emissions, $Q_{d}^{t}$ also minimizes the latter.

Proposition 2 The buyer's optimal order quantity resulting from the decentralized model is given by

$$
Q_{d}^{*}=\sqrt{\frac{2\left(K_{b}+t_{b} f_{b}\right) D}{h_{b}+t_{b} g_{b}}} .
$$

Observe that as $t_{b}$ increases, the cost-minimizing order quantity $Q_{d}^{*}$ approaches the emission optimal order quantity $Q_{d}^{t}$. We use Proposition 2 to find the minimum average annual cost of the buyer under the decentralized model and present it in the following corollary.

Corollary 2 The average annual cost of the buyer under the optimal solution of the decentralized model is given by

$$
B C\left(Q_{d}^{*}\right)=\sqrt{2\left(K_{b}+t_{b} f_{b}\right) D\left(h_{b}+t_{b} g_{b}\right)}+\left(c+t_{b} e_{b}\right) D .
$$

Similarly, the vendor's average annual cost under the decentralized model $\left(V C\left(Q_{d}^{*}\right)\right)$ can be found by plugging $Q_{d}^{*}$ into Expression (13). Also, the total average annual cost of the system under the decentralized model is $T C\left(Q_{d}^{*}\right)=B C\left(Q_{d}^{*}\right)+V C\left(Q_{d}^{*}\right)$.

The order quantity that minimizes the average annual taxes of the system (i.e., $Q_{c}^{t}$ ) is given by

$$
Q_{c}^{t}=\sqrt{\frac{2\left(t_{b} f_{b}+t_{v} f_{v}\right) D}{t_{b} g_{b}+\frac{t_{v} g_{v} D}{P}}} .
$$

Note that $Q_{c}^{t}$ also minimizes the average annual emissions if $t_{b}=t_{v}$.

Theorem 2 The buyer's optimal order quantity resulting from the centralized model is given by

$$
Q_{c}^{*}=\sqrt{\frac{2\left(K_{b}+K_{v}+t_{b} f_{b}+t_{v} f_{v}\right) D}{h_{b}+t_{b} g_{b}+\left(h_{v}+t_{v} g_{v}\right) \frac{D}{P}}} .
$$

As $t_{v}$ gets larger, $Q_{c}^{*}$ approaches $\sqrt{\frac{2 f_{v} D}{g_{v}}}$, which is the minimizer of $V T(Q)$ (i.e., the vendor's emission optimal order quantity). Similarly, as $t_{b}$ gets larger, $Q_{c}^{*}$ approaches to $\sqrt{\frac{2 f_{b} D}{g_{b}}}$, which is the buyer's emission optimal order quantity. In the next corollary, we present the average annual cost of the system resulting from the optimal solution of the centralized model. 
Corollary 3 The total average annual cost of the system under the centralized model is given by

$$
\begin{aligned}
T C\left(Q_{c}^{*}\right)= & \sqrt{2\left(K_{b}+K_{v}+t_{b} f_{b}+t_{v} f_{v}\right) D\left[h_{b}+t_{b} g_{b}+\left(h_{v}+t_{v} g_{v}\right) \frac{D}{P}\right]} \\
& +\left(c+p_{v}+t_{b} e_{b}+t_{v} e_{v}\right) D .
\end{aligned}
$$

Similarly, the buyer's average annual cost $\left(B C\left(Q_{c}^{*}\right)\right)$ and the vendor's average annual cost $\left(V C\left(Q_{c}^{*}\right)\right)$ under the centralized model can be found by plugging $Q_{c}^{*}$ into $B C(Q)$ and in Expression (13), respectively. In the next proposition, we present a further property of $Q_{d}^{*}$ and $Q_{c}^{*}$.

Proposition $3 Q_{d}^{*} \leqslant Q_{c}^{*}$ if and only if $\frac{K_{b}+t_{b} f_{b}}{h_{b}+t_{b} g_{b}} \leqslant \frac{K_{v}+t_{v} f_{v}}{h_{v}+t_{v} g_{v}} \frac{P}{D}$.

The above proposition implies that any coordination mechanism should take into account both the case of $Q_{d}^{*}>Q_{c}^{*}$ and the case of $Q_{d}^{*}<Q_{c}^{*}$. As an example, a per unit discount of $\frac{B C\left(Q_{c}^{*}\right)-B C\left(Q_{d}^{*}\right)}{D}$ for order quantities greater than or equal to $Q_{c}^{*}$ if $Q_{d}^{*}<Q_{c}^{*}$, and less than or equal to $Q_{c}^{*}$ if $Q_{d}^{*}>Q_{c}^{*}$ would coordinate the system.

Until this point, we have taken the perspective of the buyer-vendor system in comparing the different solution approaches. We have obtained results on how the buyer's and the vendor's annual costs differ under the decentralized and centralized solutions. In the next two propositions, we take the perspective of the regulator or the government who collects taxes. We compare the average annual amount of taxes collected by the government under the decentralized and centralized solutions.

Proposition 4 Suppose $\frac{K_{b}+t_{b} f_{b}}{h_{b}+t_{b} g_{b}} \leqslant \frac{K_{v}+t_{v} f_{v}}{h_{v}+t_{v} g_{v}} \frac{P}{D}$.

(i) If $\frac{t_{b} f_{b}+t_{v} f_{v}}{t_{b} g_{b}+\frac{t_{v} g_{v} D}{P}} \leqslant \frac{K_{b}+t_{b} f_{b}}{h_{b}+t_{b} g_{b}}$, then the government collects no fewer taxes in the centralized solution than it does in the decentralized solution.

(ii) If $\frac{t_{b} f_{b}+t_{v} f_{v}}{t_{b} g_{b}+\frac{t_{v} v_{D} D}{P}} \geqslant \frac{K_{b}+K_{v}+t_{b} f_{b}+t_{v} f_{v}}{h_{b}+t_{b} g_{b}+\left(h_{v}+t_{v} g_{v}\right) \frac{D}{P}}$, then the government collects no fewer taxes in the decentralized solution than it does in the centralized solution.

Proposition 5 Suppose $\frac{K_{b}+t_{b} f_{b}}{h_{b}+t_{b} g_{b}}>\frac{K_{v}+t_{v} f_{v}}{h_{v}+t_{v} g_{v}} \frac{P}{D}$.

(i) If $\frac{t_{b} f_{b}+t_{v} f_{v}}{t_{b} g_{b}+\frac{t_{v} g_{v} D}{P}} \geqslant \frac{K_{b}+t_{b} f_{b}}{h_{b}+t_{b} g_{b}}$, then the government collects more taxes in the centralized solution than it does in the decentralized solution.

(ii) If $\frac{t_{b} f_{b}+t_{v} f_{v}}{t_{b} g_{b}+\frac{t_{v} g_{v} D}{P}} \leqslant \frac{K_{b}+K_{v}+t_{b} f_{b}+t_{v} f_{v}}{h_{b}+t_{b} g_{b}+\left(h_{v}+t_{v} g_{v}\right) \frac{D}{P}}$, then the government collects more taxes in the decentralized solution than it does in the centralized solution.

Proof The proof follows a similar structure to the proof of Proposition 4 and is omitted.

Proposition 4 and Proposition 5 imply that there are cases in which coordination of the buyervendor system may not be good from the perspective of a government or a regulator who wants to increase total annual average taxes collected. In Table 8, we present some numerical instances to illustrate our analytical results for the buyer-vendor coordination problem under the tax policy. In the last two columns of the table, we report the decentralized and the centralized optimum quantities. In Table 9, we present the buyer's, vendor's, and system's average annual taxes resulting from the decentralized and the centralized solutions to the examples in Table 8. Examples 19, 20, and 21 are to illustrate the first part of Proposition 4. 
Table 8 Numerical instances for illustrating analytical results under the tax mechanism $\left(h_{v}=1.5, c=9\right.$, $p_{v}=6, e_{b}=5$ and $e_{v}=6$ in all instances)

\begin{tabular}{lrrrrrrrllllll}
\hline $\begin{array}{l}\text { Example } \\
\text { index }\end{array}$ & $D$ & $P$ & $K_{b}$ & $K_{v}$ & $h_{b}$ & $f_{b}$ & $f_{v}$ & $g_{b}$ & $g_{v}$ & $t_{b}$ & $t_{v}$ & $Q_{d}^{*}$ & $Q_{c}^{*}$ \\
\hline 19 & 90 & 100 & 200 & 600 & 2 & 30 & 60 & 0.2 & 0.75 & 2 & 3 & 139.642 & 180.043 \\
20 & 50 & 100 & 700 & 600 & 2 & 60 & 90 & 1 & 0.75 & 2 & 3 & 143.178 & 169.605 \\
21 & 50 & 100 & 700 & 600 & 2 & 60 & 90 & 1 & 0.6 & 2 & 3 & 143.178 & 172.949 \\
22 & 50 & 100 & 40 & 60 & 2 & 70 & 90 & 1 & 0.75 & 2 & 3 & 67.082 & 93.171 \\
23 & 90 & 100 & 200 & 600 & 2 & 100 & 120 & 0.15 & 0.75 & 2 & 3 & 176.930 & 207.693 \\
24 & 50 & 100 & 40 & 60 & 2 & 30 & 120 & 3 & 2 & 2 & 3 & 35.355 & 66.525 \\
25 & 40 & 60 & 400 & 60 & 2 & 300 & 60 & 0.6 & 0.2 & 4 & 2 & 170.561 & 158.523 \\
26 & 500 & 600 & 800 & 60 & 1.7 & 750 & 310 & 1 & 0.75 & 2 & 3 & 788.430 & 694.299 \\
27 & 550 & 600 & 450 & 70 & 2 & 300 & 80 & 1.7 & 0.2 & 4 & 2 & 454.148 & 442.915 \\
28 & 50 & 60 & 900 & 60 & 1.7 & 60 & 90 & 1 & 0.75 & 2 & 3 & 166.034 & 140.642 \\
29 & 40 & 90 & 800 & 60 & 1.7 & 60 & 90 & 1 & 0.7 & 2 & 3 & 141.039 & 137.361 \\
30 & 500 & 600 & 800 & 60 & 1.7 & 400 & 90 & 1 & 0.75 & 2 & 3 & 657.596 & 531.774 \\
\hline & & & & & & & & & & & & &
\end{tabular}

Table 9 Average annual taxes resulting from the decentralized and the centralized solutions of the instances in Table 8

\begin{tabular}{lllllll}
\hline $\begin{array}{l}\text { Example } \\
\text { index }\end{array}$ & $B T\left(Q_{d}^{*}\right)$ & $V T\left(Q_{d}^{*}\right)$ & $T T\left(Q_{d}^{*}\right)$ & $B T\left(Q_{c}^{*}\right)$ & $V T\left(Q_{c}^{*}\right)$ & $T T\left(Q_{c}^{*}\right)$ \\
\hline 19 & 966.599 & 1877.399 & 2843.997 & 966.001 & 1892.272 & 2858.274 \\
20 & 685.084 & 1074.826 & 1759.91 & 704.982 & 1075 & 1779.981 \\
21 & 685.084 & 1058.718 & 1743.802 & 707.642 & 1055.885 & 1763.526 \\
22 & 671.432 & 1138.98 & 1810.412 & 668.302 & 1097.303 & 1765.605 \\
23 & 1028.275 & 1982.265 & 3010.539 & 1017.82 & 1986.289 & 3004.109 \\
24 & 690.919 & 1462.15 & 2153.069 & 744.670 & 1270.363 & 2015.033 \\
25 & 1286.098 & 530.884 & 1816.982 & 1293.023 & 531.416 & 1824.439 \\
26 & 6739.688 & $10,328.93$ & $17,068.62$ & 6774.525 & $10,320.65$ & $17,095.17$ \\
27 & $13,997.37$ & 6877.03 & $20,874.4$ & $13,996.04$ & 6879.885 & $20,875.92$ \\
28 & 702.172 & 1136.966 & 1839.138 & 683.304 & 1127.84 & 1811.144 \\
29 & 575.072 & 862.393 & 1437.465 & 572.305 & 862.727 & 1435.032 \\
30 & 6265.872 & 9281.789 & $16,087.66$ & 6283.973 & 9752.405 & $16,036.38$ \\
\hline
\end{tabular}

As it can be observed from Table 9, in these examples, the government collects more taxes in the centralized solution than it does in the decentralized solution (i.e., $T T\left(Q_{c}^{*}\right)>T T\left(Q_{d}^{*}\right)$ ). These examples differ in how the individual parties' average annual taxes change in the two solutions. For example, in Example 19, while $B T\left(Q_{d}^{*}\right)>B T\left(Q_{c}^{*}\right)$ and $V T\left(Q_{d}^{*}\right)<$ $V T\left(Q_{c}^{*}\right)$, in Example 20, we have $B T\left(Q_{d}^{*}\right)<B T\left(Q_{c}^{*}\right)$ and $V T\left(Q_{d}^{*}\right)<V T\left(Q_{c}^{*}\right)$. The next three examples (Examples 22, 23, 24) illustrate the second part of Proposition 4. In these examples, the government collects more taxes in the decentralized solution than it does in the centralized solution. Likewise, Examples 25, 26, and 27 illustrate the first part of Proposition 5. As evident in Table 9, in these examples, the government collects more taxes in the centralized solution. Finally, the second part of Proposition 5 is illustrated with 
Examples 28, 29, and 30, in which the government collects more average annual taxes in the decentralized solution.

We would like to note that, in Table 8 , the instances in which $T T\left(Q_{c}^{*}\right)>T T\left(Q_{d}^{*}\right)$ coincide with the cases where coordination is not good for the environment. On the other hand, instances with $T T\left(Q_{c}^{*}\right)<T T\left(Q_{d}^{*}\right)$ are illustrative of the cases in which coordination is good for the environment.

\section{Conclusion}

There is growing recognition of the potential damage of global climate change caused by human activities. Reducing greenhouse gases through some environmental regulations is possible; however, these measures impose costs on the economy, and the efficiency of different policies in the long run is uncertain. In this paper, we investigated the impact of supply chain coordination on environmental measures under two emission-regulation policies: cap-andtrade and tax. We performed our analysis over a buyer-vendor system facing deterministic demand in the infinite horizon. Our findings show that how the buyer and the vendor behave in terms of the contractual agreements they engage in has a significant effect on the resulting emissions under both policies. We conclude that in general, supply chain coordination may or may not be good for the environment, depending on the circumstances, as opposed to having no coordination under a specific policy. Furthermore, the impact of coordinated decisions in comparison to independent decisions depends on the parties' particular production/inventoryrelated parameters, which means that coordination among firms is a source of unpredictability for the policy maker in designing regulations. In case of cap-and-trade policy, one exception was when the vendor's fixed replenishment cost is extremely high. In our experimentation, we consistently observed that in such cases, coordination between the parties results in more system emissions than the uncoordinated solution does.

We also explored the added flexibility of the cap-and-trade policy for firms to share their carbon credits and we proposed novel coordination mechanisms based on carbon credit sharing. This flexibility has the potential to reduce supply chain costs even further under coordination but may sometimes contribute to higher carbon emissions. Supply chain coordination is an important aid for companies in reducing overall system costs, and carbon credit sharing as part of coordination mechanisms may help companies in reducing the cost of compliance to the cap-and-trade policy. Our results show that whether this comes at the expense of increased carbon emissions in comparison to coordination with no carbon credit sharing, again, depends on the particular parameters of the buyer and the vendor. This result suggests that the benefits of carbon credit sharing in terms of costs should be weighed against a possible increase in carbon emissions, and the policy maker should carefully determine the terms for the private transfer of carbon credits among firms.

Our review of the production/inventory models in the operations research and the management science literature revealed that number of studies considering environmental policies within the context of different problems in multi-echelon settings is limited. We would like to note that our paper is the first one to study coordination in a setting where multiple parties in the supply chain are subject to environmental policies. Furthermore, our modeling for the cap-and-trade policy allows for the purchasing price of unit carbon allowance to be larger than its selling price. The difference in carbon trading prices leads to challenging optimization problems under both independent and integrated decisions. A contribution of this paper is to propose finite-time exact solution procedures for these problems. In our modeling for 
the tax policy, we also aimed for a generalization by allowing the manufacturer's and the retailer's carbon tax rates to be different, which may happen if the parties are in different industries or in different geographical locations. A future work could be to study other problems such as facility location, transportation mode selection, etc. under the environmental policies modeled herein. We considered a deterministic-demand production-inventory setting with a lot-for-lot policy in place. It is also worthwhile to extend the questions of interest and the analysis in this paper to more complex settings by modeling different dispatch policies or stochasticity of demand.

In case of cap-and-trade policy, our consideration of the difference between the selling and purchasing prices of unit carbon allowance led us to some novel coordination mechanisms based on carbon credit sharing (i.e., carbon-credit sharing along with fixed payments, or carbon credit sharing along with quantity discounts). In case of tax policy, we showed that classical quantity discounts can be used for channel coordination. Our study assumed that retail price of the item is fixed and demand is independent of the retail price. Weng (1995) showed that when the retail price is a decision variable and demand is dependent on the retail price, a quantity discount policy is not sufficient for coordination. A further generalization of our study could be to consider a dependency between demand and retail price, which we believe may necessitate the use of different coordination mechanisms.

In this paper, we also obtained some results which can be helpful for a policy maker in designing environmental regulations. Specifically, in Corollary 1, we showed how the retailer's order quantity changes with his/her cap and how the change depends on the retailer's parameters (i.e., fixed cost and fixed emissions at each ordering, cost rate and emissions rate related to inventory holding). In Propositions 4 and 5, we provided a comparison of the taxes the government collects in case of centralized and decentralized decision-making between the buyer and the vendor, based on a characterization of their parameters. Our objective in this paper was to provide a thorough analysis for the cap-and-trade policy and the tax policy individually. A comparison of these policies within the context of coordination remains a future research. This comparison may investigate how the total emissions change after coordination under a cap-and-trade policy versus under a tax policy. However, we believe obtaining general results requires an extensive numerical study, and the problem instances should be generated carefully to consider equivalent cap-and-trade and tax policies. That is, under the appropriate parameters of the cap-and-trade policy and the corresponding tax policy, the average annual costs and the emissions should be similar in the decentralized solution for a fair comparison.

In analyzing the impact of the coordinated solution on total emissions, we defined a measure which we referred to as $R$ in the paper (ratio of average annual system emissions resulting from the optimal solution of the centralized model to that of the decentralized model). We showed that there are instances of the problem under which $R>1$ for both the cap-and-trade and the tax policies. The objective functions of the centralized and decentralized models were cost minimization. Therefore, our proposed coordinated solutions aimed for mechanisms by which the manufacturer induces the buyer to order the centralized quantity while having no worse costs than his/her decentralized solution would lead to. As a different and more environmental solution, the integrated model can be studied under the constraint $R \leq 1$ in search for dispatch quantities that have better (not necessarily best) system costs with lesser average annual emissions than the decentralized model does. New mechanisms can then be designed for the retailer to order this environmental quantity. 


\section{Appendix}

\section{Proof of Lemma 1}

For any order quantity $Q$, the amount of traded carbon credits by the buyer is $X_{b}(Q)=$ $C_{b}-\frac{f_{b} D}{Q}-\frac{g_{b} Q}{2}-e_{b} D$. Observe that $\hat{Q}_{d}$ minimizes $\frac{f_{b} D}{Q}+\frac{g_{b} Q}{2}$ with a minimum function value $\sqrt{2 f_{b} g_{b} D}$. That is,

$$
\frac{f_{b} D}{Q}+\frac{g_{b} Q}{2} \geq \sqrt{2 f_{b} g_{b} D}
$$

for all $Q \geq 0$. This implies

$$
X_{b}(Q) \leq C_{b}-e_{b} D-\sqrt{2 f_{b} g_{b} D} .
$$

Given that $\left(C_{b}-e_{b} D\right) \leq \sqrt{2 g_{b} f_{b} D}$, it turns out that $X_{b}(Q) \leq 0$ for all $Q \geq 0$. That is, the retailer does not sell carbon credits at any order quantity. In this case, Expression (1) implies that the retailer's inventory replenishment problem reduces to minimizing $B C_{1}\left(Q, X_{b}(Q)\right)$ over $Q \geq 0$. As given by Expression (15), $Q_{d 1}^{*}$ is the optimal solution of this problem.

\section{Development of the other results for the Proof of Theorem 1}

Lemma 2 The buyer sells carbon credits (i.e., $\left.X_{b}(Q)>0\right)$ only when $\left(C_{b}-e_{b} D\right)>$ $\sqrt{2 g_{b} f_{b} D}$ and $Q_{1}<Q<Q_{2}$.

Proof From Lemma 1, we know that if $\left(C_{b}-e_{b} D\right) \leq \sqrt{2 g_{b} f_{b} D}$, then the buyer does not sell carbon credits. Therefore, selling carbon credits is possible only when $\left(C_{b}-e_{b} D\right)>$ $\sqrt{2 g_{b} f_{b} D}$. Furthermore, under this condition, $X_{b}(Q)>0$ should be satisfied. $X_{b}(Q)=$ $C_{b}-\frac{f_{b} D}{Q}-\frac{g_{b} Q}{2}-e_{b} D>0$ holds for order quantities $Q$ such that $Q_{1}<Q<Q_{2}$. Note that, as $\left(C_{b}-e_{b} D\right)>\sqrt{2 g_{b} f_{b} D}$, both $Q_{1}$ and $Q_{2}$ are defined and $Q_{1}<Q_{2}$.

Lemma 2 implies that in addition to the case of $\left(C_{b}-e_{b} D\right) \leq \sqrt{2 g_{b} f_{b} D}$ suggested by Lemma 1, there are two cases in which the retailer does not sell carbon credits: if $\left(C_{b}-e_{b} D\right)>$ $\sqrt{2 g_{b} f_{b} D}$ and $Q \leq Q_{1}$, or if $\left(C_{b}-e_{b} D\right)>\sqrt{2 g_{b} f_{b} D}$ and $Q \geq Q_{2}$.

Lemma 3 Depending on how $f_{b} h_{b}$ compares to $K_{b} g_{b}$, the following ordinal relations exist between $Q_{d 1}^{*}$ and $Q_{d 2}^{*}$ :

- If $f_{b} h_{b}>K_{b} g_{b}$, then $Q_{d 1}^{*}>Q_{d 2}^{*}$.

- If $f_{b} h_{b}=K_{b} g_{b}$, then $Q_{d 1}^{*}=Q_{d 2}^{*}$.

- If $f_{b} h_{b}<K_{b} g_{b}$, then $Q_{d 1}^{*}<Q_{d 2}^{*}$.

Proof We will prove the first part of the lemma. The proofs of the remaining two parts are similar.

Since $p_{c}^{b} \geq p_{c}^{s}, f_{b} h_{b}>K_{b} g_{b}$ implies that $\left(p_{c}^{b}-p_{c}^{s}\right) f_{b} h_{b}>\left(p_{c}^{b}-p_{c}^{s}\right) K_{b} g_{b}$. Adding $K_{b} h_{b}+p_{c}^{b} p_{c}^{s} f_{b} g_{b}$ to both sides of this inequality, and after some rearrangement of terms, we have

$$
\left(K_{b}+p_{c}^{b} f_{b}\right)\left(h_{b}+p_{c}^{s} g_{b}\right)>\left(K_{b}+p_{c}^{s} f_{b}\right)\left(h_{b}+p_{c}^{b} g_{b}\right) .
$$

The above expression can be rewritten as

$$
\frac{\left(K_{b}+p_{c}^{b} f_{b}\right)}{\left(h_{b}+p_{c}^{b} g_{b}\right)}>\frac{\left(K_{b}+p_{c}^{s} f_{b}\right)}{\left(h_{b}+p_{c}^{s} g_{b}\right)},
$$


which further implies

$$
\sqrt{\frac{2\left(K_{b}+p_{c}^{b} f_{b}\right) D}{\left(h_{b}+p_{c}^{b} g_{b}\right)}}>\sqrt{\frac{2\left(K_{b}+p_{c}^{s} f_{b}\right) D}{\left(h_{b}+p_{c}^{s} g_{b}\right)}} .
$$

Observe that the left-hand side of the above inequality is $Q_{d 1}^{*}$ and the right-hand side is $Q_{d 2}^{*}$, and therefore, $Q_{d 1}^{*}>Q_{d 2}^{*}$.

In the next lemma, we present further properties of the retailer's problem in the case of $\left(C_{b}-e_{b} D\right)>\sqrt{2 g_{b} f_{b} D}$.

Lemma 4 When $\left(C_{b}-e_{b} D\right)>\sqrt{2 g_{b} f_{b} D}$, the following cases cannot be observed.

- $Q_{1}<Q_{2} \leq Q_{d 2}^{*} \leq Q_{d 1}^{*}$.

- $Q_{d 1}^{*} \leq Q_{d 2}^{*} \leq Q_{1}<Q_{2}$.

Proof Let us start with the first part of the lemma. Using Expression (17) and Expression (19), $Q_{2} \leq Q_{d 2}^{*}$ implies that

$$
\frac{C_{b}-e_{b} D+\sqrt{\left(C_{b}-e_{b} D\right)^{2}-2 g_{b} f_{b} D}}{g_{b}} \leq \sqrt{\frac{2\left(K_{b}+p_{c}^{s} f_{b}\right) D}{h_{b}+p_{c}^{s} g_{b}}} .
$$

Since $\left(C_{b}-e_{b} D\right)>\sqrt{2 g_{b} f_{b} D}$, the left-hand side is positive. Therefore, taking the square of both sides leads to

$$
\frac{\left(C_{b}-e_{b} D\right)^{2}+\left(C_{b}-e_{b} D\right) \sqrt{\left(C_{b}-e_{b} D\right)^{2}-2 g_{b} f_{b} D}-g_{b} f_{b} D}{g_{b}} \leq \frac{\left(K_{b} g_{b}+p_{c}^{s} f_{b} g_{b}\right) D}{h_{b}+p_{c}^{s} g_{b}} .
$$

Due to Lemma 3, we know that having $Q_{d 2}^{*} \leq Q_{d 1}^{*}$ is possible only when $f_{b} h_{b} \geq K_{b} g_{b}$, which implies

$$
\frac{\left(f_{b} h_{b}+p_{c}^{s} f_{b} g_{b}\right) D}{h_{b}+p_{c}^{s} g_{b}} \geq \frac{\left(K_{b} g_{b}+p_{c}^{s} f_{b} g_{b}\right) D}{h_{b}+p_{c}^{s} g_{b}} .
$$

Combining the last two inequalities, we obtain

$$
\begin{aligned}
& \frac{\left(C_{b}-e_{b} D\right)^{2}+\left(C_{b}-e_{b} D\right) \sqrt{\left(C_{b}-e_{b} D\right)^{2}-2 g_{b} f_{b} D}-g_{b} f_{b} D}{g_{b}} \\
& \leq \frac{\left(f_{b} h_{b}+p_{c}^{s} f_{b} g_{b}\right) D}{h_{b}+p_{c}^{s} g_{b}}=f_{b} D .
\end{aligned}
$$

Multiplying both sides of the above expression by $g_{b}$ and after some rearrangement of terms, it follows that

$$
\left(C_{b}-e_{b} D\right)^{2}-2 g_{b} f_{b} D \leq-\left(C_{b}-e_{b} D\right) \sqrt{\left(C_{b}-e_{b} D\right)^{2}-2 g_{b} f_{b} D} .
$$

Recall that, $Q_{1}$ and $Q_{2}$ were formed by considering the positive square root of the discriminant in $X_{b}(0)$, and $Q_{2}$ was defined as the larger root. Since $\left(C_{b}-e_{b} D\right)>\sqrt{2 g_{b} f_{b} D}$, the above inequality cannot hold for the positive square root of $\left(C_{b}-e_{b} D\right)^{2}-2 g_{b} f_{b} D$. Therefore, we cannot have $Q_{1}<Q_{2} \leq Q_{d 2}^{*} \leq Q_{d 1}^{*}$.

Now, let us continue with the second part of the lemma. Using Expression (17) and Expression (18), $Q_{d 2}^{*} \leq Q_{1}$ implies that

$$
\sqrt{\frac{2\left(K_{b}+p_{c}^{s} f_{b}\right) D}{h_{b}+p_{c}^{s} g_{b}}} \leq \frac{C_{b}-e_{b} D-\sqrt{\left(C_{b}-e_{b} D\right)^{2}-2 g_{b} f_{b} D}}{g_{b}} .
$$


Taking the square of both sides of this inequality leads to

$$
\frac{\left(K_{b}+p_{c}^{s} f_{b}\right) D}{h_{b}+p_{c}^{s} g_{b}} \leq \frac{\left(C_{b}-e_{b} D\right)^{2}-\left(C_{b}-e_{b} D\right) \sqrt{\left(C_{b}-e_{b} D\right)^{2}-2 g_{b} f_{b} D}-g_{b} f_{b} D}{\left(g_{b}\right)^{2}},
$$

which is equivalent to

$$
\frac{\left(K_{b} g_{b}+p_{c}^{s} f_{b} g_{b}\right) D}{h_{b}+p_{c}^{s} g_{b}} \leq \frac{\left(C_{b}-e_{b} D\right)^{2}-\left(C_{b}-e_{b} D\right) \sqrt{\left(C_{b}-e_{b} D\right)^{2}-2 g_{b} f_{b} D}-g_{b} f_{b} D}{g_{b}} .
$$

Based on Lemma 3, having $Q_{d 2}^{*} \geq Q_{d 1}^{*}$ suggests that $f_{b} h_{b} \leq K_{b} g_{b}$, which implies

$$
\frac{\left(f_{b} h_{b}+p_{c}^{s} f_{b} g_{b}\right) D}{h_{b}+p_{c}^{s} g_{b}} \leq \frac{\left(C_{b}-e_{b} D\right)^{2}-\left(C_{b}-e_{b} D\right) \sqrt{\left(C_{b}-e_{b} D\right)^{2}-2 g_{b} f_{b} D}-g_{b} f_{b} D}{g_{b}} .
$$

Observe that the left-hand side of the above inequality reduces to $f_{b} D$. Therefore, after some rearrangement of terms, it can be rewritten as

$$
\left(C_{b}-e_{b} D\right)^{2}-2 g_{b} f_{b} D \geq\left(C_{b}-e_{b} D\right) \sqrt{\left(C_{b}-e_{b} D\right)^{2}-2 g_{b} f_{b} D} .
$$

Again, the above inequality cannot hold for the positive square root of $\left(C_{b}-e_{b} D\right)^{2}-2 g_{b} f_{b} D$. Therefore, we cannot have $Q_{d 1}^{*} \leq Q_{d 2}^{*} \leq Q_{1}<Q_{2}$.

The first part of Lemma 4 implies that when $\left(C_{b}-e_{b} D\right)>\sqrt{2 g_{b} f_{b} D}$, the case of $Q_{1}<Q_{2} \leq Q_{d 2}^{*}=Q_{d 1}^{*}$ cannot occur. Likewise, the second part implies that when $\left(C_{b}-\right.$ $\left.e_{b} D\right)>\sqrt{2 g_{b} f_{b} D}$, the case of $Q_{d 1}^{*}=Q_{d 2}^{*} \leq Q_{1}<Q_{2}$ cannot take place. Combining this result with Lemma 3 further leads to the following implication: If $\left(C_{b}-e_{b} D\right)>\sqrt{2 g_{b} f_{b} D}$ and $f_{b} h_{b}=K_{b} g_{b}$, the only possible ordering of $Q_{1}, Q_{2}, Q_{d 1}^{*}$ and $Q_{d 2}^{*}$ is $Q_{1}<Q_{d 1}^{*}=$ $Q_{d 2}^{*}<Q_{2}$, because having $\left(C_{b}-e_{b} D\right)>\sqrt{2 g_{b} f_{b} D}$ implies $Q_{2}>Q_{1}$, and it follows due to Lemma 3 and the fact that $f_{b} h_{b}=K_{b} g_{b}$ that $Q_{d 1}^{*}=Q_{d 2}^{*}$. Under these conditions, excluding the cases covered in Lemma 4 from further consideration, the only possible ordering that remains is $Q_{1}<Q_{d 1}^{*}=Q_{d 2}^{*}<Q_{2}$.

Lemma 5 If $\left(C_{b}-e_{b} D\right)>\sqrt{2 g_{b} f_{b} D}$ and $f_{b} h_{b}=K_{b} g_{b}$, then $Q_{d}^{*}=Q_{d 1}^{*}=Q_{d 2}^{*}$.

Proof Under the conditions of the lemma, the only possible ordering of $Q_{1}, Q_{2}, Q_{d 1}^{*}$, and $Q_{d 2}^{*}$ is $Q_{1}<Q_{d 1}^{*}=Q_{d 2}^{*}<Q_{2}$. To prove the lemma, we will consider three regions of $Q$ separately: $Q \leq Q_{1}, Q_{1}<Q<Q_{2}$, and $Q \geq Q_{2}$. Expression (1) and Lemma 2 together imply that if $\left(C_{b}-e_{b} D\right)>\sqrt{2 g_{b} f_{b} D}$, for order quantities $Q$ such that $Q_{1}<Q<Q_{2}$, we have $B C\left(Q, X_{b}(Q)\right)=B C_{2}\left(Q, X_{b}(Q)\right)$; for order quantities $Q$ such that $Q \leq Q_{1}$, we have $B C\left(Q, X_{b}(Q)\right)=B C_{1}\left(Q, X_{b}(Q)\right)$; for order quantities $Q$ such that $Q \geq Q_{2}$, we have $B C\left(Q, X_{b}(Q)\right)=B C_{1}\left(Q, X_{b}(Q)\right)$.

Let us start with $Q$ such that $Q_{1}<Q<Q_{2}$ and $Q \neq Q_{d 2}^{*}$. Since $Q_{d 2}^{*}$ is the unique minimizer of $B C_{2}\left(Q, X_{b}(Q)\right)$ and $B C\left(Q, X_{b}(Q)\right)=B C_{2}\left(Q, X_{b}(Q)\right)$, it follows that

$$
\begin{aligned}
B C\left(Q, X_{b}(Q)\right)= & B C_{2}\left(Q, X_{b}(Q)\right)>B C_{2}\left(Q_{d 2}^{*}, X_{b}\left(Q_{d 2}^{*}\right)\right)=B C\left(Q_{d 2}^{*}, X_{b}\left(Q_{d 2}^{*}\right)\right), \\
& \forall Q \text { s.t. } Q_{1}<Q<Q_{2} \text { and } Q \neq Q_{d 2}^{*} .
\end{aligned}
$$

Now, let us continue with $Q \leq Q_{1}$. Recall that at $Q_{1}$, we have $B C_{1}\left(Q_{1}, X_{b}\left(Q_{1}\right)\right)=$ $B C_{2}\left(Q_{1}, X_{b}\left(Q_{1}\right)\right)$. Since $B C_{1}\left(Q, X_{b}(Q)\right)$ is a strictly convex function with a unique minimizer $Q_{d 1}^{*}$, and $Q \leq Q_{1}<Q_{d 1}^{*}$, it follows that

$$
B C_{1}\left(Q, X_{b}(Q)\right) \geq B C_{1}\left(Q_{1}, X_{b}\left(Q_{1}\right)\right)=B C_{2}\left(Q_{1}, X_{b}\left(Q_{1}\right)\right) .
$$


Using the fact that $B C_{2}\left(Q, X_{b}(Q)\right)$ is a strictly convex function with a unique minimizer $Q_{d 2}^{*}$, and $Q_{1} \neq Q_{d 2}^{*}$, we further have

$$
B C_{2}\left(Q_{1}, X_{b}\left(Q_{1}\right)\right)>B C_{2}\left(Q_{d 2}^{*}, X_{b}\left(Q_{d 2}^{*}\right)\right) .
$$

Combining the last two inequalities leads to

$$
B C_{1}\left(Q, X_{b}(Q)\right)>B C_{2}\left(Q_{d 2}^{*}, X_{b}\left(Q_{d 2}^{*}\right)\right),
$$

which is equivalent to

$$
B C\left(Q, X_{b}(Q)\right)>B C\left(Q_{d 2}^{*}, X_{b}\left(Q_{d 2}^{*}\right)\right), \quad \forall Q \text { s.t. } Q \leq Q_{1} .
$$

Finally, let us consider order quantities $Q$ such that $Q \geq Q_{2}$. Recall that at $Q_{2}$, we have $B C_{1}\left(Q_{2}, X_{b}\left(Q_{2}\right)\right)=B C_{2}\left(Q_{2}, X_{b}\left(Q_{2}\right)\right)$. Since $B C_{1}\left(Q, X_{b}(Q)\right)$ is a strictly convex function with a unique minimizer $Q_{d 1}^{*}$, and $Q_{d 1}^{*}<Q_{2} \leq Q$, it follows that

$$
B C_{1}\left(Q, X_{b}(Q)\right) \geq B C_{1}\left(Q_{2}, X_{b}\left(Q_{2}\right)\right)=B C_{2}\left(Q_{2}, X_{b}\left(Q_{2}\right)\right) .
$$

Using the fact that $B C_{2}\left(Q, X_{b}(Q)\right)$ is a strictly convex function with a unique minimizer $Q_{d 2}^{*}$, and $Q_{2} \neq Q_{d 2}^{*}$, we further have

$$
B C_{2}\left(Q_{2}, X_{b}\left(Q_{2}\right)\right)>B C_{2}\left(Q_{d 2}^{*}, X_{b}\left(Q_{d 2}^{*}\right)\right) .
$$

Combining the last two inequalities leads to

$$
B C_{1}\left(Q, X_{b}(Q)\right)>B C_{2}\left(Q_{d 2}^{*}, X_{b}\left(Q_{d 2}^{*}\right)\right),
$$

which, is also equivalent to

$$
B C\left(Q, X_{b}(Q)\right)>B C\left(Q_{d 2}^{*}, X_{b}\left(Q_{d 2}^{*}\right)\right), \quad \forall Q \text { s.t. } Q \geq Q_{2} .
$$

Based on Expressions (33), (34), and (35), we conclude that $Q^{*}=Q_{d 2}^{*}$.

Lemma 1 and Lemma 5 constitute parts of our solution algorithm for the retailer's decentralized replenishment problem. Lemma 1 suggests the solution in the case of $\left(C_{b}-e_{b} D\right) \leq$ $\sqrt{2 g_{b} f_{b} D}$, and Lemma 5 provides the solution in the case of $\left(C_{b}-e_{b} D\right)>\sqrt{2 g_{b} f_{b} D}$ and $f_{b} h_{b}=K_{b} g_{b}$. At this point, there is one more case to be considered, that is, $\left(C_{b}-e_{b} D\right)>\sqrt{2 g_{b} f_{b} D}$ and $f_{b} h_{b} \neq K_{b} g_{b}$. Before proceeding with a detailed analysis of this case, let us present another result that applies to the case of $\left(C_{b}-e_{b} D\right)>\sqrt{2 g_{b} f_{b} D}$ in general.

Lemma 6 When $\left(C_{b}-e_{b} D\right)>\sqrt{2 g_{b} f_{b} D}$, we have $B C_{1}\left(Q, X_{b}(Q)\right) \leq B C_{2}\left(Q, X_{b}(Q)\right)$ for all $Q$ such that $Q_{1} \leq Q \leq Q_{2}$, and $B C_{1}\left(Q, X_{b}(Q)\right)>B C_{2}\left(Q, X_{b}(Q)\right)$ for all $Q$ such that $Q<Q_{1}$ or $Q>Q_{2}$.

Proof Recall that $X_{b}(Q)=C_{b}-\frac{f_{b} D}{Q}-\frac{g_{b} Q}{2}-e_{b} D$, and $X_{b}(Q)=0$ when $Q=Q_{1}$ and $Q=Q_{2}$. Furthermore, we have $X_{b}(Q)>0$ for all $Q$ s.t. $Q_{1}<Q<Q_{2}$, and we have $X_{b}(Q)<0$ for all $Q$ s.t. $Q<Q_{1}$ and for all $Q$ s.t. $Q>Q_{2}$. We will show that $B C_{1}\left(Q, X_{b}(Q)\right) \leq B C_{2}\left(Q, X_{b}(Q)\right)$ if $Q \in\left[Q_{1}, Q_{2}\right]$. The proofs of the other parts of the lemma, which are omitted, follow in a similar fashion.

Since $p_{c}^{b} \geq p_{c}^{s}$, it follows that

$$
\left(p_{c}^{b}-p_{c}^{s}\right)\left(C_{b}-\frac{f_{b} D}{Q}-\frac{g_{b} Q}{2}-e_{b} D\right) \geq 0 .
$$


After adding $\frac{K_{b} D}{Q}+\frac{h_{b} Q}{2}+c D$ to both sides of the above inequality and rearranging the terms, we have

$$
\begin{aligned}
& \frac{K_{b} D}{Q}+\frac{h_{b} Q}{2}+c D-p_{c}^{b}\left(C_{b}-\frac{f_{b} D}{Q}-\frac{g_{b} Q}{2}-e_{b} D\right) \\
& \leq \frac{K_{b} D}{Q}+\frac{h_{b} Q}{2}+c D-p_{c}^{s}\left(C_{b}-\frac{f_{b} D}{Q}-\frac{g_{b} Q}{2}-e_{b} D\right),
\end{aligned}
$$

which implies $B C_{1}\left(Q, X_{b}(Q)\right) \leq B C_{2}\left(Q, X_{b}(Q)\right)$.

The above lemma will be used in the proofs of the next two results.

Lemma 7 When $\left(C_{b}-e_{b} D\right)>\sqrt{2 g_{b} f_{b} D}$ and $f_{b} h_{b}<K_{b} g_{b}$, the following orderings among $Q_{1}, Q_{2}, Q_{d 1}^{*}$, and $Q_{d 2}^{*}$ cannot take place:

- $Q_{d 1}^{*} \leq Q_{1}<Q_{2} \leq Q_{d 2}^{*}$,

- $Q_{d 1}^{*} \leq Q_{1}<Q_{d 2}^{*}<Q_{2}$, and

- $Q_{d 1}^{*}<Q_{d 2}^{*} \leq Q_{1}<Q_{2}$.

Proof We will prove the first two parts of the lemma. Note that the third part is a special case of $Q_{d 1}^{*} \leq Q_{d 2}^{*} \leq Q_{1}<Q_{2}$ and is covered in Lemma 4 .

Due to the strict convexity of $B C_{2}\left(Q, X_{b}(Q)\right)$ and the fact that $Q_{d 2}^{*}$ is its minimizer, having $Q_{1}<Q_{2} \leq Q_{d 2}^{*}$ implies

$$
B C_{2}\left(Q_{1}, X_{b}\left(Q_{1}\right)\right)>B C_{2}\left(Q_{2}, X_{b}\left(Q_{2}\right)\right) .
$$

At $Q=Q_{1}$ and $Q=Q_{2}$, we have $B C_{1}\left(Q, X_{b}(Q)\right)=B C_{2}\left(Q, X_{b}(Q)\right)$. Therefore, the above inequality is equivalent to the following:

$$
B C_{1}\left(Q_{1}, X_{b}\left(Q_{1}\right)\right)>B C_{1}\left(Q_{2}, X_{b}\left(Q_{2}\right)\right) .
$$

However, due to the strict convexity of $B C_{1}\left(Q, X_{b}(Q)\right)$ and $Q_{d 1}^{*}$ being its unique minimizer, having $Q_{d 1}^{*} \leq Q_{1}<Q_{2}$ would imply

$$
B C_{1}\left(Q_{1}, X_{b}\left(Q_{1}\right)\right)<B C_{1}\left(Q_{2}, X_{b}\left(Q_{2}\right)\right) .
$$

Expression (36) and (37) contradict, therefore, it is not possible to have $Q_{d 1}^{*} \leq Q_{1}<Q_{2} \leq$ $Q_{d 2}^{*}$.

Now, let us continue with the proof of the second part. Note that Lemma 6 and its proof imply

$$
B C_{1}\left(Q_{d 2}^{*}, X_{b}\left(Q_{d 2}^{*}\right)\right)<B C_{2}\left(Q_{d 2}^{*}, X_{b}\left(Q_{d 2}^{*}\right)\right)
$$

in case of $Q_{1}<Q_{d 2}^{*}<Q_{2}$. Furthermore, having $Q_{1}<Q_{d 2}^{*}$ leads to $B C_{2}\left(Q_{d 2}^{*}, X_{b}\left(Q_{d 2}^{*}\right)\right)<$ $B C_{2}\left(Q_{1}, X_{b}\left(Q_{1}\right)\right)$ due to the strict convexity of $B C_{2}\left(Q, X_{b}(Q)\right)$ and the fact that $Q_{d 2}^{*}$ is its minimizer. Combining this with the above inequality implies

$$
B C_{1}\left(Q_{d 2}^{*}, X_{b}\left(Q_{d 2}^{*}\right)\right)<B C_{2}\left(Q_{1}, X_{b}\left(Q_{1}\right)\right) .
$$

At $Q=Q_{1}$, we have $B C_{2}\left(Q_{1}, X_{b}\left(Q_{1}\right)\right)=B C_{1}\left(Q_{1}, X_{b}\left(Q_{1}\right)\right)$. Therefore, the above expression is equivalent to

$$
B C_{1}\left(Q_{d 2}^{*}, X_{b}\left(Q_{d 2}^{*}\right)\right)<B C_{1}\left(Q_{1}, X_{b}\left(Q_{1}\right)\right) .
$$

However, due to the strict convexity of $B C_{1}\left(Q, X_{b}(Q)\right)$ and $Q_{d 1}^{*}$ being its unique minimizer, having $Q_{d 1}^{*} \leq Q_{1}<Q_{d 2}^{*}$ would imply

$$
B C_{1}\left(Q_{d 2}^{*}, X_{b}\left(Q_{d 2}^{*}\right)\right)>B C_{1}\left(Q_{1}, X_{b}\left(Q_{1}\right)\right) .
$$


Table 10 Numerical illustrations of Corollaries 4 and 5 given $D=50, c=12$ and $g_{b}=0.5$

\begin{tabular}{lccccccccccc}
\hline $\begin{array}{l}\text { Example } \\
\text { index }\end{array}$ & $K_{b}$ & $h_{b}$ & $f_{b}$ & $e_{b}$ & $p_{b}^{c}$ & $p_{s}^{c}$ & $C_{b}$ & $Q_{d 1}^{*}$ & $Q_{d 2}^{*}$ & $Q_{1}$ & $Q_{2}$ \\
\hline 1 & 900 & 1 & 40 & 5 & 7.5 & 6 & 300 & 158.944 & 168.819 & 55.279 & 144.721 \\
2 & 500 & 1 & 90 & 5 & 7.5 & 6 & 350 & 157.28 & 161.245 & 51.676 & 348.324 \\
3 & 900 & 1 & 40 & 5 & 7.5 & 6 & 303 & 158.944 & 168.819 & 49.114 & 162.886 \\
4 & 100 & 1.2 & 90 & 5 & 2.5 & 2 & 320 & 115.175 & 112.815 & 100 & 180 \\
5 & 40 & 3.2 & 90 & 4.5 & 2.5 & 2 & 304 & 77.169 & 72.375 & 74.549 & 241.451 \\
6 & 40 & 3.2 & 90 & 4.5 & 2.5 & 2 & 300 & 77.169 & 72.375 & 82.918 & 217.082 \\
\hline
\end{tabular}

As Expressions (38) and (39) contradict, it is not possible to have $Q_{d 1}^{*} \leq Q_{1}<Q_{d 2}^{*}$ $<Q_{2}$.

Notice that, since $\left(C_{b}-e_{b} D\right)>\sqrt{2 g_{b} f_{b} D}$ and $f_{b} h_{b}<K_{b} g_{b}$ are the two conditions of Lemma 7, two common properties of the cases considered are $Q_{1}<Q_{2}$ and $Q_{d 1}^{*}<Q_{d 2}^{*}$. Lemma 7 further leads to the result in Corollary 4.

Corollary 4 When $\left(C_{b}-e_{b} D\right)>\sqrt{2 g_{b} f_{b} D}$ and $f_{b} h_{b}<K_{b} g_{b}$, the following orderings are possible:

- $Q_{1}<Q_{2} \leq Q_{d 1}^{*}<Q_{d 2}^{*}$,

- $Q_{1}<Q_{d 1}^{*}<Q_{d 2}^{*} \leq Q_{2}$, and

- $Q_{1}<Q_{d 1}^{*}<Q_{2}<Q_{d 2}^{*}$.

Numerical instances to illustrate the cases in Corollary 4 (and Corollary 5) are presented in Table 10. The first three examples of Table 10 correspond to the different cases of the corollary in the order they are presented.

In the next lemma, we provide a similar result to Lemma 7, now for the case of $\left(C_{b}-\right.$ $\left.e_{b} D\right)>\sqrt{2 g_{b} f_{b} D}$ and $f_{b} h_{b}>K_{b} g_{b}$.

Lemma 8 When $\left(C_{b}-e_{b} D\right)>\sqrt{2 g_{b} f_{b} D}$ and $f_{b} h_{b}>K_{b} g_{b}$, the following orderings among $Q_{1}, Q_{2}, Q_{d 1}^{*}$, and $Q_{d 2}^{*}$ cannot take place:

- $Q_{d 2}^{*}<Q_{1}<Q_{2} \leq Q_{d 1}^{*}$,

- $Q_{1} \leq Q_{d 2}^{*}<Q_{2} \leq Q_{d 1}^{*}$, and

- $Q_{1}<Q_{2} \leq Q_{d 2}^{*}<Q_{d 1}^{*}$.

Proof Similar to the proof of Lemma 7, we will prove the first two parts of the lemma. The third part is a special case of $Q_{1}<Q_{2} \leq Q_{d 2}^{*} \leq Q_{d 1}^{*}$ and is covered in Lemma 4.

Let us assume that the ordering in the first part of the lemma takes place. Due to Lemma 6, having $Q_{2} \leq Q_{d 1}^{*}$ implies $B C_{1}\left(Q_{d 1}^{*}, X_{b}\left(Q_{d 1}^{*}\right)\right) \geq B C_{2}\left(Q_{d 1}^{*}, X_{b}\left(Q_{d 1}^{*}\right)\right)$. Furthermore, it follows from the strict convexity of $B C_{2}\left(Q, X_{b}(Q)\right)$ that having $Q_{d 2}^{*}<Q_{1}<Q_{2} \leq Q_{d 1}^{*}$ leads to

$$
B C_{2}\left(Q_{d 1}^{*}, X_{b}\left(Q_{d 1}^{*}\right)\right) \geq B C_{2}\left(Q_{2}, X_{b}\left(Q_{2}\right)\right)>B C_{2}\left(Q_{1}, X_{b}\left(Q_{1}\right)\right),
$$

and hence, $B C_{1}\left(Q_{d 1}^{*}, X_{b}\left(Q_{d 1}^{*}\right)\right)>B C_{2}\left(Q_{1}, X_{b}\left(Q_{1}\right)\right)$. At $Q=Q_{1}$, we have $B C_{2}\left(Q, X_{b}\right.$ $(Q))=B C_{1}\left(Q, X_{b}(Q)\right)$. Therefore, if the ordering is true, as it is assumed, it would follow that $B C_{1}\left(Q_{d 1}^{*}, X_{b}\left(Q_{d 1}^{*}\right)\right)>B C_{1}\left(Q_{1}, X_{b}\left(Q_{1}\right)\right)$, which contradicts with $Q_{d 1}^{*}$ being the minimizer of $B C_{1}\left(Q, X_{b}(Q)\right)$. Therefore, it is not possible to have $Q_{d 2}^{*}<Q_{1}<Q_{2} \leq Q_{d 1}^{*}$. 
Let us continue with the proof of the second part by assuming that there exists an instance with this ordering. Due to Lemma 6, having $Q_{1} \leq Q_{d 2}^{*}<Q_{2}$ implies $B C_{2}\left(Q_{d 2}^{*}, X_{b}\left(Q_{d 2}^{*}\right)\right) \geq B C_{1}\left(Q_{d 2}^{*}, X_{b}\left(Q_{d 2}^{*}\right)\right)$. Furthermore, it follows from the strict convexity of $B C_{1}\left(Q, X_{b}(Q)\right)$ that having $Q_{d 2}^{*}<Q_{2} \leq Q_{d 1}^{*}$ leads to

$$
B C_{1}\left(Q_{d 2}^{*}, X_{b}\left(Q_{d 2}^{*}\right)\right)>B C_{1}\left(Q_{2}, X_{b}\left(Q_{2}\right)\right) \geq B C_{1}\left(Q_{d 1}^{*}, X_{b}\left(Q_{d 1}^{*}\right)\right),
$$

and hence, $B C_{2}\left(Q_{d 2}^{*}, X_{b}\left(Q_{d 2}^{*}\right)\right)>B C_{1}\left(Q_{d 1}^{*}, X_{b}\left(Q_{d 1}^{*}\right)\right)$. Using Lemma 6 once again and the fact that $Q_{d 1}^{*} \geq Q_{2}$, we must have $B C_{1}\left(Q_{d 1}^{*}, X_{b}\left(Q_{d 1}^{*}\right)\right) \geq B C_{2}\left(Q_{d 1}^{*}, X_{b}\left(Q_{d 1}^{*}\right)\right)$, which would imply $B C_{2}\left(Q_{d 2}^{*}, X_{b}\left(Q_{d 2}^{*}\right)\right)>B C_{2}\left(Q_{d 1}^{*}, X_{b}\left(Q_{d 1}^{*}\right)\right)$. However, this contradicts with the fact that $Q_{d 2}^{*}$ is the minimizer of $B C_{2}\left(Q, X_{b}(Q)\right)$. Therefore, it is not possible to have $Q_{1} \leq Q_{d 2}^{*}<Q_{2} \leq Q_{d 1}^{*}$.

Note that under the two conditions of Lemma 8, two common properties of the cases considered are $Q_{1}<Q_{2}$ and $Q_{d 1}^{*}>Q_{d 2}^{*}$. Lemma 8 further leads to the result in the next corollary.

Corollary 5 When $\left(C_{b}-e_{b} D\right)>\sqrt{2 g_{b} f_{b} D}$ and $f_{b} h_{b}>K_{b} g_{b}$, the following orderings are possible:

- $Q_{1} \leq Q_{d 2}^{*}<Q_{d 1}^{*}<Q_{2}$,

- $Q_{d 2}^{*}<Q_{1}<Q_{d 1}^{*}<Q_{2}$, and

- $Q_{d 2}^{*}<Q_{d 1}^{*} \leq Q_{1}<Q_{2}$.

Numerical instances to illustrate the cases in Corollary 5 are also presented in Table 10. The last three examples of Table 10 correspond to the different cases of the corollary in the order they are presented.

\section{Proof of Theorem 1}

The proof will follow based on considering the cases presented in Lemma 1, Lemma 5, Corollary 4, and Corollary 5.

Case 1: $\left(C_{b}-e_{b} D\right) \leq \sqrt{2 g_{b} f_{b} D}$

It follows due to Lemma 1 that in this case $Q_{d}^{*}=Q_{d 1}^{*}$.

Case 2: $\left(C_{b}-e_{b} D\right)>\sqrt{2 g_{b} f_{b} D}$

We have the following three subcases $\left(f_{b} h_{b}=K_{b} g_{b}, f_{b} h_{b}<K_{b} g_{b}\right.$, and $\left.f_{b} h_{b}>K_{b} g_{b}\right)$ :

Case 2.1: $\left(C_{b}-e_{b} D\right)>\sqrt{2 g_{b} f_{b} D}$ and $f_{b} h_{b}=K_{b} g_{b}$

It follows due to Lemma 5 that in this case $Q_{d}^{*}=Q_{d 2}^{*}$.

Case 2.2: $\left(C_{b}-e_{b} D\right)>\sqrt{2 g_{b} f_{b} D}$ and $f_{b} h_{b}<K_{b} g_{b}$

Corollary 4 implies the following three subcases: $Q_{1}<Q_{2} \leq Q_{d 1}^{*}<Q_{d 2}^{*}, Q_{1}<Q_{d 1}^{*}<$ $Q_{d 2}^{*} \leq Q_{2}$, and $Q_{1}<Q_{d 1}^{*}<Q_{2}<Q_{d 2}^{*}$. We present a detailed proof for the first subcase. Since the proofs of the other subcases are similar, we present sketches of the proofs for those.

- Case 2.2.1: $Q_{1}<Q_{2} \leq Q_{d 1}^{*}<Q_{d 2}^{*}$

Note that the subcase of $Q_{1}<Q_{2} \leq Q_{d 1}^{*}<Q_{d 2}^{*}$ is distinguished from the other two by the fact that $Q_{2} \leq Q_{d 1}^{*}$. The proof will follow by considering three different regions of $Q\left(Q>Q_{2}, Q_{1} \leq Q \leq Q_{2}, Q<Q_{1}\right)$, and in each case by showing that $B C\left(Q_{d 1}^{*}, X_{b}\left(Q_{d 1}^{*}\right)\right) \leq B C\left(Q, X_{b}(Q)\right)$. Let us start with $Q$ values such that $Q>Q_{2}$. Expression (1) and Lemma 2 imply that $B C\left(Q, X_{b}(Q)\right)=$ $B C_{1}\left(Q, X_{b}(Q)\right)$. By definition, $Q_{d 1}^{*}$ is the minimizer of $B C_{1}\left(Q, X_{b}(Q)\right)$, therefore, $B C_{1}\left(Q, X_{b}(Q)\right) \geq B C_{1}\left(Q_{d 1}^{*}, X_{b}\left(Q_{d 1}^{*}\right)\right)$. Since $Q_{d 1}^{*}$ is also in the region of $Q$ values considered (i.e., $\left.Q_{d 1}^{*} \geq Q_{2}\right)$, this, in turn, is equivalent to $B C\left(Q, X_{b}(Q)\right) \geq$ 
$B C\left(Q_{d 1}^{*}, X_{b}\left(Q_{d 1}^{*}\right)\right)$. Now, let us consider $Q$ values such that $Q_{1} \leq Q \leq Q_{2}$. Expression (1) and Lemma 2 imply that $B C\left(Q, X_{b}(Q)\right)=B C_{2}\left(Q, X_{b}(Q)\right)$. Since $B C_{2}\left(Q, X_{b}(Q)\right)$ is a strictly convex function with a unique minimizer $Q_{d 2}^{*}$ and $Q<Q_{d 2}^{*}, B C_{2}\left(Q, X_{b}(Q)\right)$, and hence $B C\left(Q, X_{b}(Q)\right)$, is decreasing in this region. Therefore, $B C\left(Q, X_{b}(Q)\right) \geq B C\left(Q_{2}, X_{b}\left(Q_{2}\right)\right)$ for all $Q$ such that $Q_{1} \leq Q \leq Q_{2}$. Furthermore, we have $B C\left(Q_{2}, X_{b}\left(Q_{2}\right)\right)=B C_{2}\left(Q_{2}, X_{b}\left(Q_{2}\right)\right)=B C_{1}\left(Q_{2}, X_{b}\left(Q_{2}\right)\right)$ and $B C_{1}\left(Q_{2}, X_{b}\left(Q_{2}\right)\right) \geq B C_{1}\left(Q_{d 1}^{*}, X_{b}\left(Q_{d 1}^{*}\right)\right)=B C\left(Q_{d 1}^{*}, X_{b}\left(Q_{d 1}^{*}\right)\right)$. Hence, $B C\left(Q, X_{b}(Q)\right) \geq B C\left(Q_{d 1}^{*}, X_{b}\left(Q_{d 1}^{*}\right)\right)$. Finally, let us consider $Q$ values such that $Q<Q_{1}$. Again, due to Expression (1) and Lemma 2, we know that $B C\left(Q, X_{b}(Q)\right)=$ $B C_{1}\left(Q, X_{b}(Q)\right)$. Since $B C_{1}\left(Q, X_{b}(Q)\right)$ is a strictly convex function with a unique minimizer $Q_{d 1}^{*}$ and $Q<Q_{d 1}^{*}, B C_{1}\left(Q, X_{b}(Q)\right)$, and hence $B C\left(Q, X_{b}(Q)\right)$, is decreasing in this region. Therefore, $B C\left(Q, X_{b}(Q)\right)>B C\left(Q_{1}, X_{b}\left(Q_{1}\right)\right)$ for all $Q$ such that $Q<Q_{1}$. We have discussed above that $B C\left(Q, X_{b}(Q)\right)$ is decreasing over $Q_{1} \leq Q \leq Q_{2}$, hence $B C\left(Q_{1}, X_{b}\left(Q_{1}\right)\right)>B C\left(Q_{2}, X_{b}\left(Q_{2}\right)\right)$. Combining the last two results implies $B C\left(Q, X_{b}(Q)\right)>B C\left(Q_{2}, X_{b}\left(Q_{2}\right)\right)$. We have also argued above that $B C\left(Q_{2}, X_{b}\left(Q_{2}\right)\right) \geq B C\left(Q_{d 1}^{*}, X_{b}\left(Q_{d 1}^{*}\right)\right)$. Therefore, we conclude $B C\left(Q, X_{b}(Q)\right)>$ $B C\left(Q_{d 1}^{*}, X_{b}\left(Q_{d 1}^{*}\right)\right)$.

- Case 2.2.2: $Q_{1}<Q_{d 1}^{*}<Q_{d 2}^{*} \leq Q_{2}$

We have $B C\left(Q, X_{b}(Q)\right) \geq B C\left(Q_{d 2}^{*}, X_{b}\left(Q_{d 2}^{*}\right)\right)$ for all $Q \in\left[Q_{1}, Q_{2}\right]$, because, $Q_{1}<Q_{d 2}^{*} \leq Q_{2}$ and $B C\left(Q, X_{b}(Q)\right)=B C_{2}\left(Q, X_{b}(Q)\right)$ in this region of $Q$ values. Next, we use the facts that $B C\left(Q, X_{b}(Q)\right)=B C_{1}\left(Q, X_{b}(Q)\right)$ for all $Q \in$ $\left(Q_{2}, \infty\right), B C_{1}\left(Q, X_{b}(Q)\right)$ is increasing in this region, and $B C_{1}\left(Q_{2}, X_{b}\left(Q_{2}\right)\right)=$ $B C_{2}\left(Q_{2}, X_{b}\left(Q_{2}\right)\right)$ to conclude that $B C\left(Q, X_{b}(Q)\right)>B C\left(Q_{2}, X_{b}\left(Q_{2}\right)\right)$. This further implies $B C\left(Q, X_{b}(Q)\right)>B C\left(Q_{d 2}^{*}, X_{b}\left(Q_{d 2}^{*}\right)\right)$ for all $Q \in\left(Q_{2}, \infty\right)$. Finally, using the facts that $B C\left(Q, X_{b}(Q)\right)=B C_{1}\left(Q, X_{b}(Q)\right)$ for all $Q$ such that $Q<Q_{1}, B C_{1}\left(Q, X_{b}(Q)\right)$ is decreasing in this region, and $B C_{1}\left(Q_{1}, X_{b}\left(Q_{1}\right)\right)=$ $B C_{2}\left(Q_{1}, X_{b}\left(Q_{1}\right)\right)$ to conclude that $B C\left(Q, X_{b}(Q)\right)>B C\left(Q_{1}, X_{b}\left(Q_{1}\right)\right)$. This further implies $B C\left(Q, X_{b}(Q)\right)>B C\left(Q_{d 2}^{*}, X_{b}\left(Q_{d 2}^{*}\right)\right)$ for all $Q$ such that $Q<Q_{1}$.

- Case 2.2.3: $Q_{1}<Q_{d 1}^{*}<Q_{2}<Q_{d 2}^{*}$

We have $B C\left(Q, X_{b}(Q)\right) \geq B C\left(Q_{2}, X_{b}\left(Q_{2}\right)\right)$ for all $Q \in\left[Q_{1}, Q_{2}\right]$, because, $B C\left(Q, X_{b}(Q)\right)=B C_{2}\left(Q, X_{b}(Q)\right)$ and $Q_{1}<Q_{2}<Q_{d 2}^{*}$ (implying that $B C_{2}\left(Q, X_{b}(Q)\right)$ is decreasing in this region of $Q$ values). Next, we use the facts that $B C\left(Q, X_{b}(Q)\right)=B C_{1}\left(Q, X_{b}(Q)\right)$ for all $Q \in\left(Q_{2}, \infty\right)$ and $Q_{d 1}^{*}<Q_{2}$ (implying that $B C_{1}\left(Q, X_{b}(Q)\right)$ is increasing in this region) to conclude that $B C\left(Q, X_{b}(Q)\right)>$ $B C\left(Q_{2}, X_{b}\left(Q_{2}\right)\right)$. Finally, using the facts that $B C\left(Q, X_{b}(Q)\right)=B C_{1}\left(Q, X_{b}(Q)\right)$ for all $Q$ such that $Q<Q_{1}$, and $Q_{1}<Q_{d 1}^{*}$ (implying that $B C_{1}\left(Q, X_{b}(Q)\right)$ is decreasing in this region), we conclude that $B C\left(Q, X_{b}(Q)\right)>B C\left(Q_{1}, X_{b}\left(Q_{1}\right)\right)$. Combining this with the fact that $B C\left(Q_{1}, X_{b}\left(Q_{1}\right)\right)>B C\left(Q_{2}, X_{b}\left(Q_{2}\right)\right)$ further leads to $B C\left(Q, X_{b}(Q)\right)>B C\left(Q_{2}, X_{b}\left(Q_{2}\right)\right)$ for all $Q$ such that $Q<Q_{1}$.

Case 2.2: $\left(C_{b}-e_{b} D\right)>\sqrt{2 g_{b} f_{b} D}$ and $f_{b} h_{b}>K_{b} g_{b}$

Corollary 5 implies the following three subcases: $Q_{d 2}^{*}<Q_{1}<Q_{2} \leq Q_{d 1}^{*}, Q_{1} \leq Q_{d 2}^{*}<$ $Q_{2} \leq Q_{d 1}^{*}, Q_{1}<Q_{2} \leq Q_{d 2}^{*}<Q_{d 1}^{*}$. A detailed proof will be omitted for this case as it follows by analyzing the different subcases, as in the proof of Case 2.1.

\section{Proof of Proposition 1}

Suppose $C_{b}-e_{b} D \leq \sqrt{2 g_{b} f_{b} D}$. Using Expression (1) and Lemma 1, $B C\left(Q, X_{b}(Q)\right)=$ $B C_{1}\left(Q, X_{b}(Q)\right)$ for all $Q$. Since $B C_{1}\left(Q, X_{b}(Q)\right)$ is a strictly convex function of $Q$, $B C\left(Q, X_{b}(Q)\right)$ is also a strictly convex function of $Q$. 
Now, suppose that $C_{b}-e_{b} D>\sqrt{2 g_{b} f_{b} D}$. The proof will follow by showing that $B C\left(\alpha Q_{a}+(1-\alpha) Q_{b}, X_{b}\left(\alpha Q_{a}+(1-\alpha) Q_{b}\right)\right)<\alpha B C\left(Q_{a}, X_{b}\left(Q_{a}\right)\right)+(1-\alpha) B C\left(Q_{b}, X_{b}\right.$ $\left.\left(Q_{b}\right)\right)$ for all $Q_{a} \geqslant 0, Q_{b} \geqslant 0\left(Q_{a} \neq Q_{b}\right)$, and $\alpha \epsilon(0,1)$. First, observe from Expression (1), Lemma 2, and Lemma 6 that $B C\left(Q, X_{b}(Q)\right)=\max \left\{B C_{1}\left(Q, X_{b}(Q)\right), B C_{2}\left(Q, X_{b}(Q)\right)\right\}$ when $C_{b}-e_{b} D>\sqrt{2 g_{b} f_{b} D}$. Since $B C_{1}\left(Q, X_{b}(Q)\right)$ and $B C_{2}\left(Q, X_{b}(Q)\right)$ are strictly convex functions of $Q$, it follows that $B C_{1}\left(\alpha Q_{a}+(1-\alpha) Q_{b}, X_{b}\left(\alpha Q_{a}+(1-\right.\right.$ $\left.\left.\alpha) Q_{b}\right)\right)<\alpha B C_{1}\left(Q_{a}, X_{b}\left(Q_{a}\right)\right)+(1-\alpha) B C_{1}\left(Q_{b}, X_{b}\left(Q_{b}\right)\right)$ and $B C_{2}\left(\alpha Q_{a}+(1-\right.$ $\left.\alpha) Q_{b}, X_{b}\left(\alpha Q_{a}+(1-\alpha) Q_{b}\right)\right)<\alpha B C_{2}\left(Q_{a}, X_{b}\left(Q_{a}\right)\right)+(1-\alpha) B C_{2}\left(Q_{b}, X_{b}\left(Q_{b}\right)\right)$ for all $Q_{a} \geqslant 0, Q_{b} \geqslant 0\left(Q_{a} \neq Q_{b}\right)$ and $\alpha \epsilon(0,1)$. Combining this with $B C\left(Q, X_{b}(Q)\right)=$ $\max \left\{B C_{1}\left(Q, X_{b}(Q)\right), B C_{2}\left(Q, X_{b}(Q)\right)\right\}$ leads to $B C_{1}\left(\alpha Q_{a}+(1-\alpha) Q_{b}, X_{b}\left(\alpha Q_{a}+\right.\right.$ $\left.\left.(1-\alpha) Q_{b}\right)\right)<\alpha B C\left(Q_{a}, X_{b}\left(Q_{a}\right)\right)+(1-\alpha) B C\left(Q_{b}, X_{b}\left(Q_{b}\right)\right)$ and $B C_{2}\left(\alpha Q_{a}+(1-\right.$ $\left.\alpha) Q_{b}, X_{b}\left(\alpha Q_{a}+(1-\alpha) Q_{b}\right)\right)<\alpha B C\left(Q_{a}, X_{b}\left(Q_{a}\right)\right)+(1-\alpha) B C\left(Q_{b}, X_{b}\left(Q_{b}\right)\right)$. Hence, $\max \left\{B C_{1}\left(\alpha Q_{a}+(1-\alpha) Q_{b}, X_{b}\left(\alpha Q_{a}+(1-\alpha) Q_{b}\right)\right), B C_{2}\left(\alpha Q_{a}+(1-\alpha) Q_{b}, X_{b}\left(\alpha Q_{a}+\right.\right.\right.$ $\left.\left.\left.(1-\alpha) Q_{b}\right)\right)\right\}<\alpha B C\left(Q_{a}, X_{b}\left(Q_{a}\right)\right)+(1-\alpha) B C\left(Q_{b}, X_{b}\left(Q_{b}\right)\right)$. Note that the left-hand side of this inequality is $B C\left(Q, X_{b}(Q)\right)$. Thus, $B C\left(Q, X_{b}(Q)\right)$ is also a strictly convex function of $Q$ if $C_{b}-e_{b} D>\sqrt{2 g_{b} f_{b} D}$.

\section{Proof of Proposition 3}

Using Equations (28) and (31), we have $Q_{d}^{*} \leqslant Q_{c}^{*}$ if and only if

$$
\sqrt{\frac{2\left(K_{b}+t_{b} f_{b}\right) D}{h_{b}+t_{b} g_{b}}} \leqslant \sqrt{\frac{2\left(K_{b}+K_{v}+t_{b} f_{b}+t_{v} f_{v}\right) D}{h_{b}+t_{b} g_{b}+\left(h_{v}+t_{v} g_{v}\right) \frac{D}{P}}} .
$$

Taking the square of both sides leads to

$$
\frac{2\left(K_{b}+t_{b} f_{b}\right) D}{h_{b}+t_{b} g_{b}} \leqslant \frac{2\left(K_{b}+K_{v}+t_{b} f_{b}+t_{v} f_{v}\right) D}{h_{b}+t_{b} g_{b}+\left(h_{v}+t_{v} g_{v}\right) \frac{D}{P}} .
$$

This, in turn, implies

$$
\begin{aligned}
& K_{b} h_{b}+K_{b} t_{b} g_{b}+K_{b} h_{v} \frac{D}{P}+K_{b} t_{v} g_{v} \frac{D}{P}+t_{b} f_{b} h_{b}+t_{b}^{2} f_{b} g_{b}+h_{v} t_{b} f_{b} \frac{D}{P}+t_{b} f_{b} t_{v} g_{v} \frac{D}{P} \\
& \leqslant K_{b} h_{b}+K_{v} h_{b}+h_{b} t_{b} f_{b}+h_{b} t_{v} f_{v}+K_{b} t_{b} g_{b}+K_{v} t_{b} g_{b}+t_{b}^{2} f_{b} g_{b}+t_{b} g_{b} t_{v} g_{v} .
\end{aligned}
$$

After some cancellations and rearrangement of terms, we get

$$
\left(K_{b}+t_{b} f_{b}\right)\left(h_{v}+t_{v} g_{v}\right) \frac{D}{P} \leqslant\left(K_{v}+t_{v} f_{v}\right)\left(h_{b}+t_{b} g_{b}\right) .
$$

This results in

$$
\frac{K_{b}+t_{b} f_{b}}{h_{b}+t_{b} g_{b}} \leqslant \frac{K_{v}+t_{v} f_{v}}{h_{v}+t_{v} g_{v}} \frac{P}{D}
$$

\section{Proof of Proposition 4}

(i) It follows from Proposition 3 that $\frac{K_{b}+t_{b} f_{b}}{h_{b}+t_{b} g_{b}} \leqslant \frac{K_{v}+t_{v} f_{v}}{h_{v}+t_{v} g_{v}} \frac{P}{D}$ is equivalent to $Q_{d}^{*} \leq Q_{c}^{*}$. Multiplying both sides of the inequality $\frac{t_{b} f_{b}+t_{v} f_{v}}{t_{b} g_{b}+\frac{t_{v} g_{P} D}{P}} \leqslant \frac{K_{b}+t_{b} f_{b}}{h_{b}+t_{b} g_{b}}$ with $2 D$ and taking the square root of both sides, we obtain

$$
\sqrt{\frac{2\left(t_{b} f_{b}+t_{v} f_{v}\right) D}{t_{b} g_{b}+\frac{t_{v} g_{v} D}{P}}} \leqslant \sqrt{\frac{2\left(K_{b}+t_{b} f_{b}\right) D}{h_{b}+t_{b} g_{b}}},
$$


which implies $Q_{c}^{t} \leq Q_{d}^{*}$. Combining this result with the fact that $Q_{d}^{*} \leq Q_{c}^{*}$ implies that $Q_{c}^{t} \leq Q_{d}^{*} \leq Q_{c}^{*}$. Since $T T(Q)$ is a strictly convex function, this implies that $T T\left(Q_{c}^{*}\right) \geq T T\left(Q_{d}^{*}\right)$. That is, in the centralized solution, the government collects at least the same amount of taxes as it collects in the decentralized solution.

(ii) Again, due to Proposition 3, we know that $\frac{K_{b}+t_{b} f_{b}}{h_{b}+t_{b} g_{b}} \leqslant \frac{K_{v}+t_{v} f_{v}}{h_{v}+t_{v} g_{v}} \frac{P}{D}$ implies $Q_{d}^{*} \leq Q_{c}^{*}$. Multiplying both sides of the inequality $\frac{t_{b} f_{b}+t_{v} f_{v}}{t_{b} g_{b}+\frac{t_{v} g_{v} D}{P}} \geqslant \frac{K_{b}+K_{v}+t_{b} f_{b}+t_{v} f_{v}}{h_{b}+t_{b} g_{b}+\left(h_{v}+t_{v} g_{v}\right) \frac{D}{P}}$ with $2 D$ and taking the square root of both sides, we obtain

$$
\sqrt{\frac{2\left(t_{b} f_{b}+t_{v} f_{v}\right) D}{t_{b} g_{b}+\frac{t_{v} g_{v} D}{P}}} \geqslant \sqrt{\frac{2\left(K_{b}+K_{v}+t_{b} f_{b}+t_{v} f_{v}\right) D}{h_{b}+t_{b} g_{b}+\left(h_{v}+t_{v} g_{v}\right) \frac{D}{P}}},
$$

which is equivalent to $Q_{c}^{t} \geq Q_{c}^{*}$. Combining this result with the fact that $Q_{d}^{*} \leq Q_{c}^{*}$ implies $Q_{c}^{t} \geq Q_{c}^{*} \geq Q_{d}^{*}$. Since $T T(Q)$ is a strictly convex function, this implies $T T\left(Q_{d}^{*}\right) \geq T T\left(Q_{c}^{*}\right)$. That is, in the decentralized solution, the government collects at least the same amount of taxes as it collects in the centralized solution.

\section{References}

Banerjee, A., \& Burton, S. (1994). Coordinated vs. independent inventory replenishment policies for a vendor and multiple buyers. International Journal of Production Economics, 35(1-3), 215-222.

Benjaafar, S., Li, Y., \& Daskin, M. (2013). Carbon footprint and the management of supply chains: Insights from simple models. IEEE Transactions on Automation Science and Engineering, 10(1), 99-116.

Bonney, M., \& Jaber, M. Y. (2011). Environmentally responsible inventory models: Non-classical models for a non-classical era. International Journal of Production Economics, 133(1), 43-53.

Bouchery, Y., Ghaffari, A., Jemai, Z., \& Dallery, Y. (2011). Adjust or invest: What is the best option to green a supply chain? Tech. rep., Laboratoire Genie Industriel, Ecole Centrale Paris.

Center for Climate and Energy Solutions. (2013). Options and considerations for a federal carbon tax. Accessed February 2014. http://www.c2es.org/publications/options-considerations-federal-carbon-tax.

Center for Climate and Energy Solutions. (2014). California cap-and-trade program summary. Accessed November 2014. http://www.c2es.org/docUploads/calif-cap-trade-01-14.pdf.

Chan, C. K., Lee, Y. C. E., \& Campbell, J. F. (2013). Environmental performance-impacts of vendor-buyer coordination. International Journal of Production Economics, 145(2), 683-695.

Chen, X., Benjaafar, S., \& Elomri, A. (2013). The carbon-constrained EOQ. Operations Research Letters, 41(2), 172-179.

Cropper, M. L., \& Oates, W. E. (1992). Environmental economics: A survey. Journal of Economic Literature, 30(2), 675-740.

Dong, C., Shen, B., Chow, P.-S., Yang, L., \& Ng, C. T. (2014). Sustainability investment under cap-and-trade regulation. Annals of Operations Research. doi:10.1007/s10479-013-1514-1.

Du, S., Ma, F., Fu, Z., Zhu, L., \& Zhang, J. (2011). Game-theoretic analysis for an emission-dependent supply chain in a 'cap-and-trade' system. Annals of Operations Research. doi:10.1007/s10479-011-0964-6.

Hoen, K., Tan, T., Fransoo, J., \& Houtum, G. (2014). Effect of carbon emission regulations on transport mode selection under stochastic demand. Flexible Services and Manufacturing Journal, 26(1-2), 170-195.

Hua, G., Cheng, T. C. E., \& Wang, S. (2011). Managing carbon footprints in inventory management. International Journal of Production Economics, 132(2), 178-185.

International Emissions Trading Assosication. (2013). The world's carbon trading markets: A case study guide to emissions trading. Accessed February 2014. http://www.ieta.org/worldscarbonmarkets.

Jaber, M. Y., Glock, C. H., \& El Saadany, A. M. A. (2013). Supply chain coordination with emissions reduction incentives. International Journal of Production Research, 51(1), 69-82.

Krass, D., Nedorezov, T., \& Ovchinnikov, A. (2013). Environmental taxes and the choice of green technology. Production and Operations Management, 22(5), 1035-1055.

Letmathe, P., \& Balakrishnan, N. (2005). Environmental considerations on the optimal product mix. European Journal of Operational Research, 167(2), 398-412.

Liu, Z., Anderson, T. D., \& Cruz, J. M. (2012). Consumer environmental awareness and competition in two-stage supply chains. European Journal of Operational Research, 218(3), 602-613. 
Lu, L. (1995). A one-vendor multi-buyer integrated inventory model. European Journal of Operational Research, 81(2), 312-323.

Nouira, I., Frein, Y., \& Hadj-Alouane, A. B. (2014). Optimization of manufacturing systems under environmental considerations for a greenness-dependent demand. International Journal of Production Economics, 150(1), 188-198.

Saadany, A. E., Jaber, M. Y., \& Bonney, M. (2011). Environmental performance measures for supply chains. Management Research Review, 34(11), 1202-1221.

Song, J., \& Leng, M. (2012). Analysis of the single-period problem under carbon emissions policies. In T.-M. Choi (Ed.), Handbook of newsvendor problems. International series in operations research and management science (Vol. 176, pp. 297-313). NewYork: Springer.

Swami, S., \& Shah, J. (2013). Channel coordination in green supply chain management. Journal of the Operational Research Society, 64(3), 336-351.

Toptal, A., Özlü, H., \& Konur, D. (2014). Joint decisions on inventory replenishment and emission reduction investment under different emission regulations. International Journal of Production Research, 52(1), 243-269.

Weng, Z. K. (1995). Channel coordination and quantity discounts. Management Science, 41(9), 1509-1522.

World Meteorological Organization. (2013a). Greenhouse gas concentrations in atmosphere reach new record. Accessed February 2014. http://www.wmo.int/pages/mediacentre/press_releases/pr_980_en.html.

World Meteorological Organization. (2013b). A WMO information note: A summary of current climate change findings and figures. Accessed February 2014. http://www.wmo.int/pages/mediacentre/factsheet/ documents/ClimateChangeInfoSheet2013-11rev5FINAL.pdf.

Zhang, B., \& Xu, L. (2013). Multi-item production planning with carbon cap and trade mechanism. International Journal of Production Economics, 144(1), 118-127. 